\title{
A Surrogate Assisted Multi-objective Approach to Social Work Planning
}

\author{
Dinh Duc Nguyen ( $\square$ nddinh76@gmail.com ) \\ Academy of Military Science and Technology https://orcid.org/0000-0003-2153-0978

\section{Long Nguyen} \\ National Defense Academy \\ Hoai Xuan Nguyen \\ Vietnam Al Academy \\ Minh Binh Tran \\ Academy of Military Science and Technology
}

\section{Research Article}

Keywords: Surrogate, Working plan , Guidance, Kriging COVID-19

Posted Date: September 3rd, 2021

DOI: https://doi.org/10.21203/rs.3.rs-698131/v1

License: (9) This work is licensed under a Creative Commons Attribution 4.0 International License. Read Full License 


\title{
A surrogate assisted multi-objective approach to social work planning
}

\section{Research on memetic computation in the application to against the COVID-19 pandemic}

\author{
Dinh Nguyen Duc • Long Nguyen • Hoai \\ Nguyen Xuan • Minh Tran Binh.
}

Received: date / Accepted: date

\begin{abstract}
Planning social tasks are essential jobs of every organization, business, and government. With increasing challenges of society, the organization and effective implementation depend on optimizing the plans of the organization and efficient operations of the professional teams in order, time, and specific requirements. In the context of the impact of the COVID-19 pandemic on social activities, developing strategies for the organization and operation of working teams in implementing disease prevention, control, and elimination are research issues that should be raised. This paper model the plan to organize and operate the social-mission working group problem with a multi-objective approach. The problem includes organizing and planning the health workforce to perform tasks in epidemic prevention and implementing guidelines of the Ministry of Health under the administration of the government. These pose a requirement to balance resources, medical equipment, and ancillary equipment to perform tasks according to different priority levels: disease prevention; vaccination; sterilization, isolation, treatment by different locations, and time to en-
\end{abstract}

Dinh Nguyen Duc

Academy of Military Science and Technology

Hanoi, Vietnam

E-mail: nddinh76@gmail.com

Long Nguyen

National Defense Academy

Hanoi, Vietnam

E-mail: longit76@gmail.com

Hoai Nguyen Xuan

Vietnam AI Academy

Hanoi, Vietnam

E-mail: nxhoai@gmail.com

Minh Tran Binh

Academy of Military Science and Technology

Hanoi, Vietnam

E-mail: minhchip79@gmail.com 
sure effectiveness. The problem is modeled by approaching the multi-objective optimization with three objectives: makespan, performance efficiency, and rate of human resource usage. We also propose a guidance technique to improve the surrogate-assisted multi-objective optimization algorithms on analyzing the factors that influence finding solutions and maintain a balance between local exploration and global exploitation. The enhanced algorithms confirm the proposed model for social tasks against the COVID-19 pandemic.

Keywords Surrogate · Working plan · Guidance · Kriging COVID-19

\section{Introduction}

The problem associated with organizational planning and inadequate social activities for working teams is a form of resource-constrained project scheduling problem (RCPSP) [14]. The organization is forced to practice a series of continuous, interdependent, and limited use of resources (including human resources, equipment, etc.) to complete a task that meets certain objectives and under specific constrains. Many problem-modeling techniques exist, but at the core of which is the construction of the objective and constraint functions. There some research in this field has been proposed.

The authors in [10] proposed a black-box model to calculate the energy efficiency of an integrated energy system, after defining the energy quality coefficient, which values the quality of various energy forms. A multi-objective planning model that considered energy efficiency and the economy was proposed for the joint planning of energy generators, storage, and networks. Here, a five-tier energy hub is modeled to deal with the multi-energy couplings. [11] presents the construction of metropolitan parks to enable the government to plan and manage urban green space, to protect the biological resources of the area, provide people with leisure and recreational places, improve the quality of life of residents, increase the economic value of neighborhoods close to metropolitan parks, and encourage environmental protection through ecotourism. The proposed design uses a multi-criteria decision-making system. A multi-objective photovoltaic power generation planning model is presented in [2], whose objectives in the operation of the distribution network are: active power losses, voltage deviation, voltage harmonic distortion, and static voltage steady index. The authors used a multi-attribution decision-making method combined with game theory to coordinate different objectives. The proposal employs a comprehensive weight model of the different objectives to present the combined effect of the photovoltaic power generation system toward distribution network. In [7], the authors proposed an approach for simultaneous optimization of distributed generation (DG) penetration level and network performance index to obtain optimal numbers, sites, and sizes of DG units. Here, two objective functions are constructed: DG penetration level and network 
performance index. Minimizing the first objective reduces the capital investment cost of a distribution network owner to integrate DG while minimizing the second objective reduces network losses and improves node voltage profile and line loading. In [1], proposed community-level energy planning with goal programming and different weighting schemes including gray weights, which represents expert judgment, was used to analyze the optimal energy supply system for a community. The model used various weighting scenarios to consider optimizing the energy system and to investigate the effects of changing decisions on outcomes and stakeholder interests. The experiments confirm that integrating maximum renewable energy supply with locally available energy resources is beneficial in energy supply cost reduction and in mitigating lifecycle environmental impacts.

Existing articles on planning that employs a multi-objective approach focuses on the objective function related to time, cost, and efficiency. However, in the planning problems of social tasks, responding to objective and subjective developments affecting the priority of the tasks is key. Therefore, consideration is given to the use of human resources, which is the basis for other contingency conditions; building adaptive plans according to the problem; organize and perform work for health working teams in the current complicated situation of the COVID-19 pandemic in countries, especially those with difficult conditions in medical personnel resources and medical preventive equipment. To make job planning more practical and enable better organizational and performance planning in social tasks, this study introduces a multi-optimization model for the social task planning problem with time-based resource constraints, the overall efficiency, and human resource to ensure high redundancy and response to social tasks that change over time.

The rest of the paper is organized as follows: Section 2 provides problem formulations, notations, and descriptions; section 3 presents the effects of guidance technique for surrogate-assisted multi-objective algorithms; section 4 presents experiments and discussion; finally, section 5 presents the conclusions.

\section{Problem formulation}

\subsection{Problem description}

A working plan for social tasks should meet some basic requirements:

First, it should consider the situation of medical forces when planning for the social task. There are different tasks, such as prevention, vaccination, disinfection, isolation, treatment, and there can be more tasks taking place at the same time. Common tasks are tied together, for example, prevention with vaccination; isolation with treatment... Therefore, each task starts only after one or more tasks have been completed

Second, each task requires two resources, human (number of people), medical equipment (number of devices), to be completed in a fixed time (duration). One or more tasks can be performed in parallel at a time, but the total re- 
source used does not exceed the total available resources, i.e., no more than the total number of people and the total amount of equipment.

Lastly, each plan is made of several sequences of tasks satisfying the above requirements, yet optimal in terms of the following:

- The total execution time of the whole plan is the lowest. In implementing social tasks, the shorter the time, the better the execution strategy is. Therefore, minimizing the execution time is a high priority requirement.

- The total performance of the whole task is the highest. This shows that prioritizing the tasks on time is more important (with higher efficiency weights).

- The ability to reserve human (or equipment) resources at a time is highest. In implementing social tasks, especially those with high social priority, such as the COVID-19 epidemic control tasks of governments, it is essential to have provision for people to complete their tasks at all times while being available to serve unexpected missions. A good plan always has competent human backups. At each time, the lower the average usage of human resources, the higher the contingency is.

Thus, the problem of planning social tasks for the health force can be expressed in the form of a multi-objective optimization problem with three objectives: Minimize the total implementation time; Maximize total performance efficiency; Minimize the average usage of human resources.

The social task implementation plan consists of a sequence of $\mathrm{n}$ different tasks and is represented by a graph $G(V, E)$, where $V$ is the set of vertices and $\mathrm{E}$ is the set of edges. $V$ consists of $n+2$ vertices, with vertex 0 starting the plan (start), vertex $n+1$ ends the plan (end) and vertices $1,2, \ldots, n$ are tasks $1,2, \ldots$ $\mathrm{n}$. Each vertex is assigned labels: inner label is the number of the task (Task ID); The above label is the time taken to complete a task (in days); The label below includes three labels, two of them in brackets representing the number of people and the amount of equipment needed to perform the task. The other label is the effective weight of the task. $E$ consists of edges representing the binding relationship between tasks. The direction of the arrow shows which task should be completed before starting another task.

An example of a plan consisting of 6 tasks $(1,2,3,4,5,6)$ is shown graphically as Fig.1 [9]. In this example, activity 4 is started only when activities 1 and 2 are finished.

\subsection{Mathematical model}

\subsubsection{Notation}

\section{Decision variables}

The health workforce's social plan will have to cover $n$ different tasks: $a=$ $\left\{a_{1}, a_{2}, \ldots, a_{n}\right\}$ with $a_{i}$ is the $i^{t h}$ task, $i=1 \rightarrow n$.

Each health force social plan will consist of a sequence of tasks that is to 

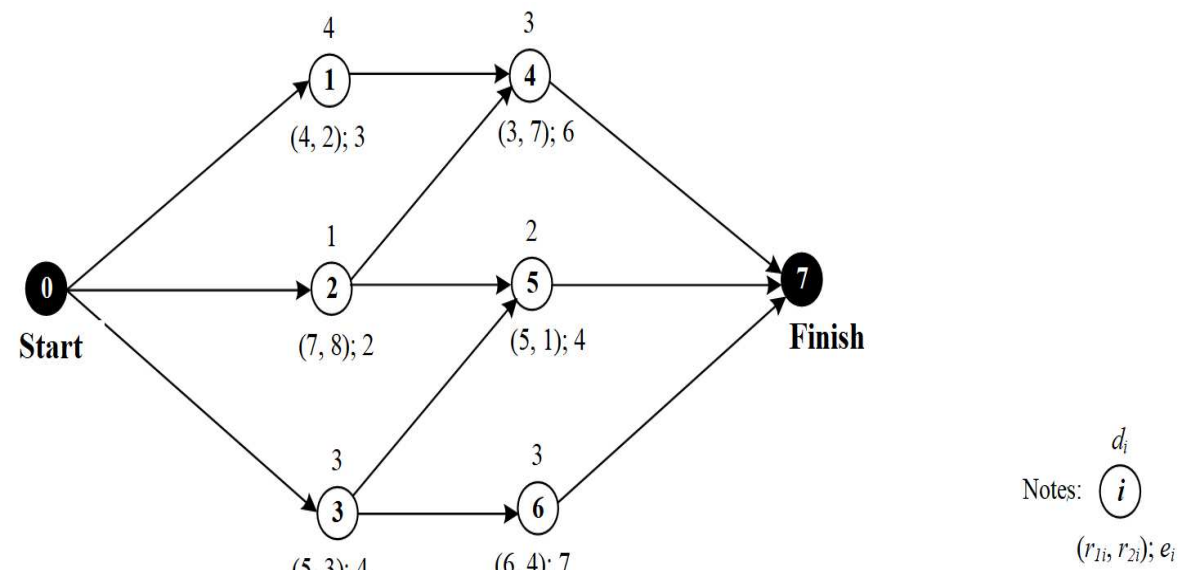

Fig. 1 An example of a graphical representation of the social plan.

permute the elements of $a$. Thus, $x$ is the permutation of $a$, which is the decision variable of the problem, $x \in A$ ( $A$ is all permutations of $a$ ), $x=$ $\left\{x_{1}, x_{2}, \ldots, x_{n}\right\}$ with $x$ is a permutation of $a$.

\section{Common parameters}

- $t$ is the time (in days), $t=1,2, \ldots T\left(T=\sum_{t=1}^{n} d_{i}\right)$.

- $R_{1}$ is the total number of people on payroll (maximum available at $t$ ).

$-R_{2}$ is the total amount of available medical equipment.

$-\alpha$ is the adjustment coefficient of the effectiveness of the task $(\alpha=0.1 \div 0.5)$.

\section{Task attributes}

$-P_{i}=$ list of finished tasks before the task $x_{i}$ starts.

$-d_{i}$ is the execution time for $x_{i}$.

- $r_{1 i}$ is the human resource required to execute $x_{i}$ (resource 1 ).

$-r_{2 i}$ is the equipment resource needed to execute $x_{i}$ (resource 2).

$-e_{i}$ is the effective performance of $x_{i}$.

- $h_{i t}$ is the status of $x_{i}$ at time $t$, the task is completed or not.

$-s t_{i}$ is the start time of the task $x_{i}$.

- et $t_{i}$ is the end time of the task $x_{i}$.

- est $t_{i}$ is the earliest start time of $x_{i}$.

\subsubsection{Objective functions}

There are three objective functions, which are formed following: Objective 1: Total time

$$
f_{1}=\max \left\{e t_{i} \mid i=1 \rightarrow n\right\}
$$

here,

$$
e t_{i}=s t_{i}+d_{i}
$$




$$
e t_{i}=\max \left\{e t_{j} \mid j \in P_{i}\right\}
$$

and

$s t_{i}=\min \left\{t \mid \sum_{j=1}^{n} r_{1 j} h_{j k} \leq R_{1} ; \sum_{j=1}^{n} r_{2 j} h_{j k} \leq R_{2} ; k \in\left[t, t+d_{i}-1\right] ; t \in\left[\right.\right.$ est $\left.\left._{i}, T-d_{i}\right]\right\}$

Objective 2: Total effectiveness

$$
f_{2}=\sum_{i=1}^{n} e_{i}(1+\alpha)^{e s t_{i}-s t_{i}}
$$

Objective 2: Average rate of use of human resources

$$
f_{3}=\frac{\sum_{t=1}^{T} \sum_{i=1}^{n} r_{1 i} h_{i t}}{\max \left\{e t_{i} \mid i=1 \rightarrow n\right\}}
$$

here,

$$
\begin{gathered}
T=\sum_{i=1}^{n} d_{i} \\
e s t_{i}=\max \left\{e t_{j} \mid j \in P_{i}\right\}, \forall i=1 \rightarrow n . \\
\max \left\{e t_{i} \mid i=1 \rightarrow n\right\} \leq T \\
\sum_{i=1}^{n} r_{1 i} h_{i t} \leq R_{1}, \forall t=1 \rightarrow T . \\
\sum_{i=1}^{n} r_{2 i} h_{i t} \leq R_{2}, \forall t=1 \rightarrow T . \\
h_{i t}=\left\{\begin{array}{l}
\text { if } x_{i} \leq s t_{i}, \forall i=1 \rightarrow n . \\
0, \quad \text { otherwise being taken at time } t .
\end{array}\right.
\end{gathered}
$$

\subsubsection{Multi-objective model}

In the social work planning problem, we need to minimize objective 1 and 3 , it also needs to maximize objective 2 . So, the problem has the following general model:

$$
\operatorname{minimize}\left\{f_{1}(x),-f_{2}(x), f_{3}(x)\right\}
$$

The first objective is to minimize the total execution time; the second objective is to maximize the total efficiency. This objective conflicts with objective 1 , which has some tasks of low efficiency but can decrease the total execution time, or some tasks that are highly efficient but increase the total execution time. The third objective is to minimize the average use of human resources. Every time, when the average use of human resources is low, the better the redundancy capacity. This objective conflicts with objective 1 , the lower the average rate of resource use, the fewer people are used at each time, the less the number of tasks performed in parallel, leading to the total execution time for the plan increases and vice versa. 


\subsubsection{An example}

The Working plan consisting of 6 tasks $a=\{1,2,3,4,5,6\}$ represented visually as Fig. 1 will be parameterized as follows:

- Parameters: $n=6 ; R_{1}=10 ; R_{2}=8 ; \alpha=0.1$.

- Make the attribute table of the $a_{i}$ tasks as follows:

A plan $x=\{1,3,6,2,4,5\}$ which is a permutation of $a$ is represented on

\begin{tabular}{|c|c|c|c|c|c|}
\hline$a_{i}$ & $d_{i}$ & $r_{1 i}$ & $r_{2 i}$ & $e_{i}$ & $P_{i}$ \\
\hline 1 & 4 & 4 & 2 & 3 & 0 \\
\hline 2 & 1 & 7 & 8 & 2 & 0 \\
\hline 3 & 3 & 5 & 3 & 4 & 0 \\
\hline 4 & 3 & 3 & 7 & 6 & 1,2 \\
\hline 5 & 2 & 5 & 1 & 4 & 2,3 \\
\hline 6 & 3 & 6 & 4 & 7 & 3 \\
\hline
\end{tabular}

Table 1 An example of the attribute table for $a_{i}$ tasks of working plan

the Gantt chart as Fig. 2:
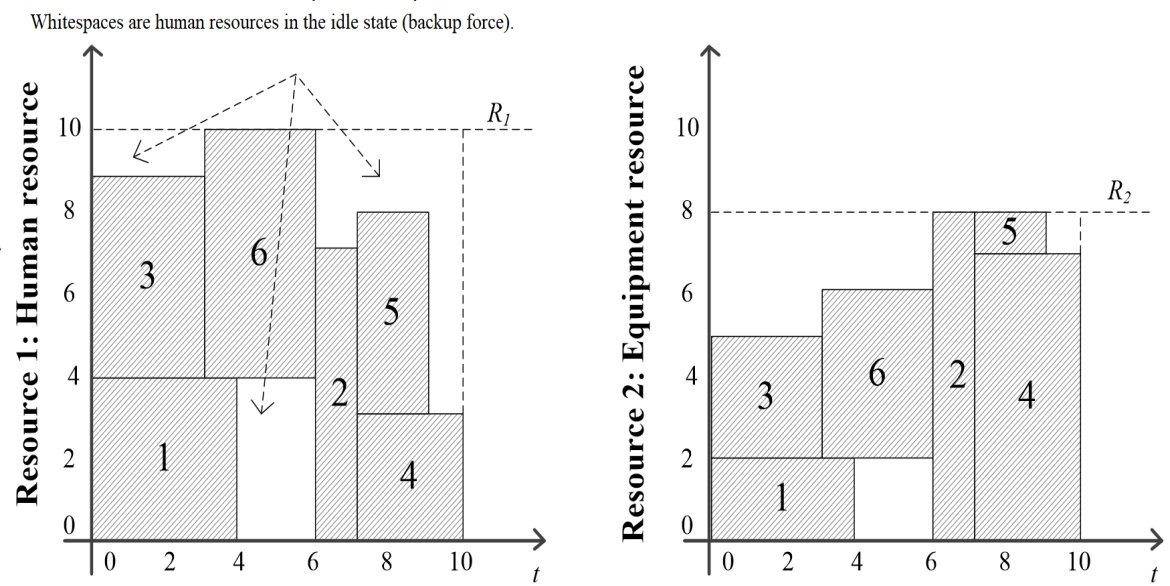

Fig. 2 An example of the plan on Gantt chart.

In fact, the problem of planning work for social forces in general, in specified features of planning for social forces in charge of COVID-19 pandemic prevention in particular, is currently very complicated. It comes from the important requirements of resource optimization, medical equipment, operational efficiency of the forces... while the force composition, diverse professional capacity, and task evolution complex cases that race with the development of the pandemic in the community. The problem will be associated with many practical constraints to the multi-objective problem model, with many complex calculations. Therefore, this is a high-cost or expensive problem. In order 
to effectively solve it and reduce the computational complexity, it is necessary to apply a multi-objective evolutionary algorithm using a surrogate model, the hot trend is applied in today's expensive problems.

However, in the application of multi-objective evolutionary algorithms using the surrogate model, there is an existing conflict that we need to have a suitable strategy, that is, the abuse of the surrogate model can make obtained results are far from the real goal of the problem (expressed in the original objective functions), the imbalance between exploration and exploitation ability can cause the evolution process to fall into local optimal regions as well as lost really good solutions in the search. To solve this problem, along with the proposal to use a multi-objective evolutionary algorithm using a surrogate model for the social force planning problem, we propose a number of guidance techniques to implement an adaptive strategy in a surrogate model mining mechanism for applied algorithms to enhance the quality of convergence and diversity and the ability to explore and exploit, so that the proposal is close to reality and can effectively be applied in the efforts of social forces fighting the current COVID-19 pandemic.

\section{A guidance technique for surrogate assisted multi-objective algorithms}

Our research analyzes factors that affect the quality of multi-objective evolutionary algorithm (MOEA) using surrogate models, hypotheses, recommendations, and guidance techniques to improve the quality of the algorithm applied to the problem of organizational and operational planning for the medical force in performing social tasks with the proposed model.

\subsection{Surrogate models}

Surrogate models are used to approximate in simulation to reduce the computational cost for expensive problems. The models are described as below: If $f(\vec{x})$ is an original fitness function of a multi-objective problem (MOP), we have $f^{\prime}(\vec{x})$ is a meta function, which is indicated as follows [6]:

$$
f^{\prime}(\vec{x})=f(\vec{x})+e(\vec{x})
$$

$e(\vec{x})$ is the approximated error. In this case, the fitness function $f(\vec{x})$ is unknown, the values (input or output) are not given. From the responses of the simulator of a chosen dataset, a surrogate is constructed, then the model generates easy representations that describe the relations between input and output variables. There are some techniques employed in surrogate models such as Kriging (KRG) [3], polynomial response surface (PRS) [12], radial basis function (RBF) [17], support vector machine (SVM) [15], artificial neural network (ANN) [8]. The common concepts associated with using surrogate models are instead of using known fitness functions, to reduce the number 
of calculations, the evaluations use surrogate functions, which are simpler. Therefore, this study also investigates the use of the original and surrogate functions with the Kriging model in solving MOEAs for expensive problems.

\subsection{Surrogate assisted multi-objective evolutionary algorithms issues}

Using the surrogate model is a relatively effective and delicate choice in solving expensive problems. The techniques commonly used in the surrogate model are KRG, PRS, RBF, SVM, ANN, etc. and can be combined with machine learning, deep learning, clustering, statistics, forecasting. However, to determine the frequency of using the surrogate function as a replacement for the original function, how to choose a reference solution, etc. creates adaptive noises. These noises are treated as transformations of the surrogate function, not the original function. This can decrease in the optimization of the algorithm. Therefore, using a surrogate model can create a noisy environment for searching MOEA. From formula 15, the interference using the surrogate model can be defined as follows:

$$
F_{\text {noise }}=f^{\prime}(\vec{x})=f(\vec{x})+e(\vec{x})
$$

Here, e is also a noisy function. For the surrogate model, the difference between the surrogate function and the original fitness function is the calculation of the noise's standard deviation and noise's distribution, respectively.

The research issue we should solve is keeping the robustness for the algorithm during the use of the surrogate model. Focusing on the performance of the algorithm through basic measurements such as GD (Generational Distance), IGD (Inverse Generational Distance) [19], we should keep the minimized values all through the search. This means we need the feasible front to be closed and spread along the Pareto optimal front in every generation. To reduce the noise in the environment, we maintain the population in the best possible ways during the search. Recently, many researchers discuss using the guidance method to drive the evolutionary process for balance in exploitation and exploration. It also maintains the convergence and diversity of the main population (obtained solutions) during the search continuously. To build up the technique, we focus on two recent surrogate-assisted multi-objective algorithms: K-RVEA [4] (using Kriging model) and CSEA [13] (using Feedforward Neural Networks - FNN). They are up-to-date surrogate-assisted multi-objective algorithms, we will work on algorithms with more investigation.

\subsubsection{K-RVEA algorithm}

a reference vector-based MOEA for solving many-objective evolutionary problems, the authors proposed a Kriging-assisted reference vector guided evolutionary algorithm (K-RVEA) [5]. In RVEA, the search process follows a set of predefined reference vectors from the direction vectors to partition the objective space into several small sub-spaces. An elitism selection strategy is 
employed inside each subspace and using an angle-penalized distance, one measures the distance of the solutions to the ideal point and the closeness of the solutions to the reference vectors. An illustration of using reference vectors is shown in the Figure 3. In K-RVEA, based on RVEA, the authors introduced

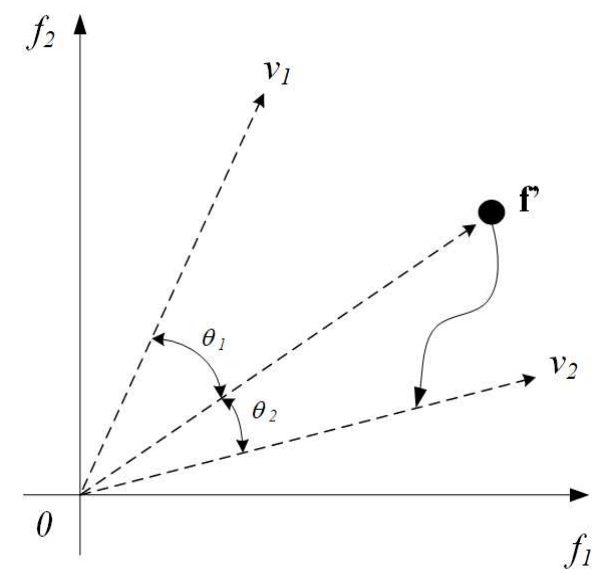

Fig. 3 An example showing how to associate an individual with a reference vector. In this example, $f^{\prime}$ is a translated objective vector, $v_{1}$ and $v_{2}$ are two unit reference vectors. $\theta_{1}$ and $\theta_{2}$ are the angles between $f^{\prime}$ and $v_{1}, v_{2}$, respectively. Since $\theta_{2}<\theta_{1}$, the individual denoted by $f^{\prime}$ is associated with reference vector $v_{2}$.

a new algorithm which has three main phases: initialization, using, and update the surrogate.

1. Initialization: algorithm uses the Latin hyper-cube sampling to generate an initial population. Two archives $A_{1}$ and $A_{2}$ are filled with individuals evaluated with the original expensive functions. A Kriging model for each objective function is built by individuals given by archive $A_{1}$.

2. Using the surrogate: In this phase, the K-RVEA uses Kriging models instead of the original functions to calculate objective function values. Kriging models are used for fitness evaluations for a prefixed number of generations without updating them.

3. Updating the surrogate: After an evolutionary search using the Kriging model for a fixed number of generations, the Kriging model will be updated. The selection of individuals to be reevaluated using the original functions, which will also be used for updating the surrogates, is essential for the performance of surrogate-assisted evolutionary algorithms (SAEAs). The authors suggested using information from the underlying evolutionary algorithm, RVEA, and uncertainty information from the Kriging model for selecting individuals to be reevaluated and for retraining the surrogate. The selected individuals are then reevaluated with the original functions and these data samples are added to both archives $A_{1}$ and $A_{2}$. The authors 
also use a reference vector-based selection strategy to keep a fixed number of individuals in the archive $A_{1}$, it will eliminate extra individuals from $A_{1}$. To assign reference vectors in various clusters see Fig4, then randomly select one data point from each cluster and eliminate the rest of the data. This technique helps the algorithm to maintain a fixed number of a diverse set of training data in $A_{1}$.

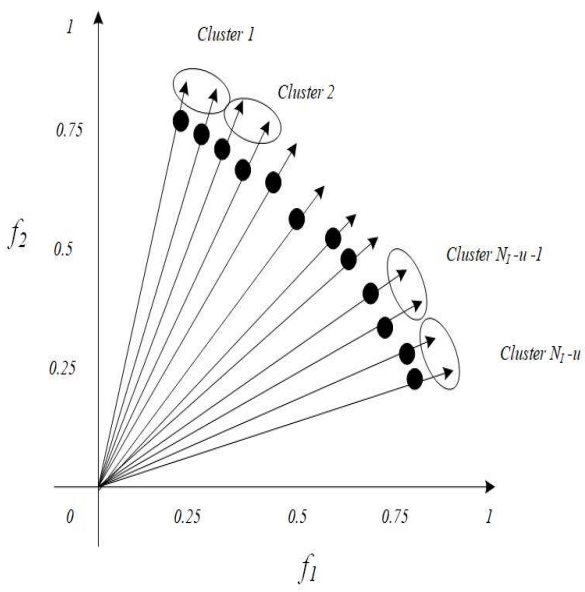

Fig. 4 Clustering of active adaptive reference vectors $V_{a}$ into a predefined number of clusters $u$.

\subsubsection{CSEA algorithm}

[13] proposed a classification-based surrogate-assisted multi-objective algorithm for expensive many-objective optimization, CSEA. A classification criterion is to divide solutions evaluated using the expensive objective functions in two categories. A surrogate is employed to learn the classification criterion, predict candidate solutions in categories, and use a selection strategy to select better-converged solutions for the evaluations. In CSEA, the primary features are the classification criterion and the surrogate management strategy.

1. Classification criterion: A classification accuracy depends on the classification criterion. Based on two types of solutions in MOPs: dominated and non-dominated, a set of reference solutions is chosen to construct the Pareto dominance boundary, which will divide all solutions into two categories.

2. The selection of reference solution: To choose a set of reference solutions to form the classification boundary, a radial space division-based 
selection strategy is employed. In this selection strategy, all solutions evaluated using the expensive fitness function are first projected into a 2dimensional radial space.

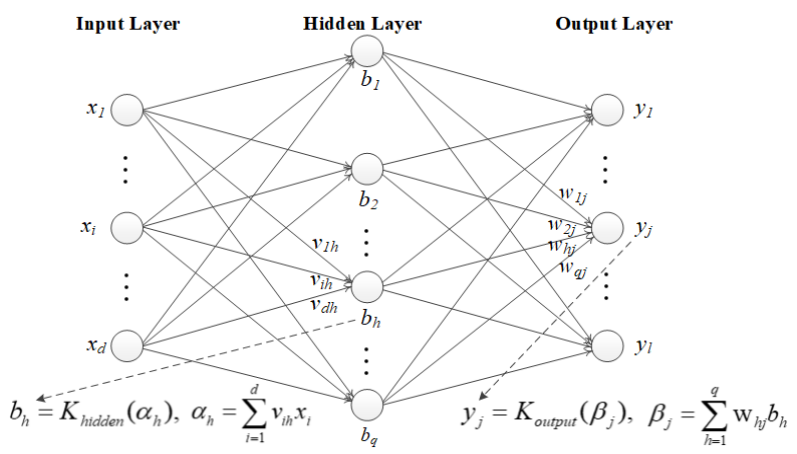

Fig. 5 Illustration of a 3-layer FNN used in the CSEA algorithm.

To manage the surrogate model, the authors design the CSEA with four basic steps:

- Initialization: Initializing the parameters ( $K$ : number of reference solutions, $g_{\max }$ : number of solutions evaluated by surrogate model) and structure of the FNN (which is one type of ANN where connections between the neurons do not form a cycle). Three main components of the FNN should be initialized: the network structure, the weights, and the activation function.

- Update: Updating the weights of the FNN with the training dataset. The authors used Levenberg-Marquardt back-propagation method to update the weights of the FNN.

- Validation: The authors used an error on test data as a measure to estimate the prediction uncertainty of the FNN. The errors are calculated by cross-validation.

- Surrogate assisted selection: In this step, some promising solutions are selected for the fitness evaluation. The selection is from the predicted performance and the reliability. The selection used the errors on test data to estimate the reliability of the prediction of the FNN. A pair of errors (Test Error Rates of two categories) forms a point located in a region of the reliability configuration, the uncertainty of the FNN. The space presents the relationship between the uncertainty of the FNN and its test errors.

The experiments in [13] confirmed that surrogates classifying solutions into dominated and non-dominated solutions are promising for solving expensive many-objective optimization problems. CSEA is an up-to-date surrogateassisted multi-objective algorithm, so we build our work on the algorithm with more investigation. 


\subsubsection{Noisy issues}

In our work, we analyzed the evolutionary process's behavior during the search with surrogate-assisted multi-objective evolutionary algorithms.

K-RVEA: states that in the first generation, the convergence of the algorithm is poor, the calculation diverse because randomly generated solutions have not evolved over generations. After that, the solution quality got better, the diversity also decreased, and spread toward the Pareto optimal front. In the last generations of the process with a specified number of generations, the quality of the population who converged was good because it processed many generations. An algorithm is assessed as good if the diversity of the population is maintained when the solutions spread evenly over the Pareto optimization front. In many analysis of the evolutionary trend of the process, to ensure a balance between convergence and diversity, it is necessary to balance the algorithm between exploration and exploitation cascade of evolution. Therefore, in the early stages, the ability to converge and end stages are needed to enhance the diversity of the population. In K-RVEA, parameters were used to determine whether the model update was fixed, to avoid making noise in the environment, which is against the robustness of the algorithm. We test the KRVEA algorithm on the class of expensive problems, with different parameters to evaluate the above comments.

We used $w_{\max }$, the number of generations before updating the Kriging model with values: 10, 20, 30, 50 and 70 with DTLZs problems [5] (DTLZs from 1 to 9 ) in 20000 evaluations. We reported the results on GD and IGD, the popular metrics to measure the performance of the algorithm. The results are shown in Tables 2, 3. Some of the behaviors of the evolution process are reported in Figures 6 to 9 . 
Table 2 GD values of K-RVEA and M-K-RVEA on DTLZs benchmark set

\begin{tabular}{|c|c|c|c|c|c|c|c|c|c|c|c|}
\hline MOEAs & $w_{\max }$ Evals & 2000 & 4000 & 6000 & 8000 & 10000 & 12000 & 14000 & 16000 & 18000 & 20000 \\
\hline & & & & & DTLZ1 & & & & & & \\
\hline & 10 & 50.7441 & & 5.6692 & 4.1103 & 3.2282 & 2.7263 & $\begin{array}{l}2.5096 \\
\end{array}$ & $\begin{array}{l}2.4711 \\
\end{array}$ & & \\
\hline & 20 & 41.9546 & 10.0818 & 10.9039 & 3.7888 & 3.7041 & 3.7041 & 4.0672 & 4.0672 & 4.0097 & 4.0097 \\
\hline K-RVEA & 30 & 39.0192 & 9.4198 & 7.2260 & 5.4170 & 3.9909 & 3.9062 & 3.9777 & 4.0149 & 3.9320 & 3.7725 \\
\hline & $\begin{array}{l}50 \\
70\end{array}$ & $\begin{array}{l}42.5498 \\
43.5096\end{array}$ & $\begin{array}{l}10.4695 \\
12.6410\end{array}$ & $\begin{array}{l}6.7609 \\
8.0107\end{array}$ & $\begin{array}{l}5.9252 \\
6.6715\end{array}$ & $\begin{array}{l}5.5349 \\
7.1815\end{array}$ & $\begin{array}{l}4.6894 \\
6.2105\end{array}$ & $\begin{array}{l}4.7492 \\
5.7701\end{array}$ & $\begin{array}{l}4.3930 \\
5.2643 \\
\end{array}$ & $\begin{array}{l}4.1622 \\
5.0035\end{array}$ & $\begin{array}{l}4.0916 \\
47697\end{array}$ \\
\hline M-K-RVEA & $\mathrm{ADC}$ & 46.6079 & 4.7267 & 3.3725 & 3.3725 & 3.3725 & 3.3725 & 3.3725 & 3.3725 & 3.3725 & 3.3725 \\
\hline & & & & & & & & & & & \\
\hline & 10 & 0.1032 & 0.0023 & 0.0014 & 0.0011 & 0.0009 & 0.0008 & 0.0008 & 0.0007 & 0.0007 & \\
\hline K-RVEA & 30 & $\begin{array}{l}0.0999 \\
0.09151\end{array}$ & 0 & 0 & 0.0008 & 0.0006 & 0.0005 & $\begin{array}{l}0.0005 \\
0.0005\end{array}$ & 0.0004 & 0.0004 & $\begin{array}{l}0.0004 \\
0.0004\end{array}$ \\
\hline & 50 & 0.1076 & 0.0015 & 0.0009 & 0.0006 & 0.0005 & 0.0005 & 0.0004 & 0.0004 & 0.0004 & 0.0004 \\
\hline & & 0.1165 & 0.0014 & 0.0010 & 0.0007 & 0.0006 & 0.0005 & 0.0005 & 0.0004 & 0.0004 & 0.0004 \\
\hline M-K-RVEA & ADC & 0.1227 & 0.0019 & 0.0011 & $\begin{array}{l}0.0008 \\
\text { DTI73 }\end{array}$ & 0.0007 & 0.0006 & 0.0006 & 0.0005 & 0.0005 & 0.0004 \\
\hline & & & & & DTLZ3 & & & & & & \\
\hline & $\begin{array}{l}10 \\
20\end{array}$ & $\begin{array}{l}183.1624 \\
171.6720\end{array}$ & $\begin{array}{l}\begin{array}{l}43.1944 \\
65.1569\end{array}\end{array}$ & $\begin{array}{l}36.1737 \\
57.1465\end{array}$ & $\begin{array}{l}33.9130 \\
47.8764\end{array}$ & $\begin{array}{l}30.8676 \\
47.3865\end{array}$ & $\begin{array}{l}29.8677 \\
42.2447\end{array}$ & $\begin{array}{l}28.4940 \\
40.1520\end{array}$ & $\begin{array}{l}30.4472 \\
38.8519\end{array}$ & $\begin{array}{l}29.3890 \\
36.7741\end{array}$ & \\
\hline K-RVEA & 30 & 220.4797 & 74.7820 & 56.873 & 52.7536 & 52.7040 & 48.8366 & $\begin{array}{l}4.15964 \\
44.596\end{array}$ & 42.4999 & 41.5517 & $\begin{array}{l}36.5496 \\
40.6848\end{array}$ \\
\hline 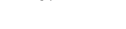 & 50 & 194.0912 & 85.5257 & 83.2413 & 70.2972 & 59.2193 & 50.7384 & 50.3928 & 49.8387 & 48.0394 & 44.5380 \\
\hline & & 151.5813 & 77.2795 & 68.4175 & 59.9071 & 51.6176 & 47.8923 & 49.5671 & 51.3997 & 48.6738 & 48.7996 \\
\hline M-K-RVEA & $\mathrm{ADC}$ & 183.1624 & 69.5647 & 54.4809 & $\frac{48.0276}{20174}$ & 47.8869 & 43.2640 & 42.0306 & 40.3582 & 39.2478 & 42.3035 \\
\hline & 10 & & & & $\begin{array}{l}\text { DTLZ4 } \\
0.0063 \\
\end{array}$ & & & & & & \\
\hline & 10 & $\begin{array}{l}0.1739 \\
0.1613\end{array}$ & $\begin{array}{l}0.0148 \\
0.0113\end{array}$ & $\begin{array}{l}0.0093 \\
0.0072\end{array}$ & $\begin{array}{l}0.0063 \\
0.0054\end{array}$ & $\begin{array}{l}0.0051 \\
0.0044\end{array}$ & $\begin{array}{l}0.0043 \\
0.0039\end{array}$ & $\begin{array}{l}0.0038 \\
0.0035\end{array}$ & $\begin{array}{l}0.0034 \\
0.0033\end{array}$ & $\begin{array}{l}0.0031 \\
0.0031\end{array}$ & $\begin{array}{l}0.0029 \\
0.0029\end{array}$ \\
\hline K-RVEA & 30 & 0.1634 & $\begin{array}{l}0.01108 \\
0.0108\end{array}$ & o. 0.0077 & & 0.0051 & 0.0044 & & & $\begin{array}{l}0.0031 \\
0.0033\end{array}$ & 0.0031 \\
\hline & 50 & 0.1841 & 0.0115 & 0.0074 & 0.0057 & 0.0048 & 0.0042 & 0.0038 & 0.0034 & 0.0031 & 0.0028 \\
\hline & 70 & 0.1564 & 0.0129 & 0.0085 & 0.0073 & 0.0063 & 0.0055 & 0.0050 & 0.0047 & 0.0045 & 0.0042 \\
\hline M-K-RVEA & ADC & 0.1564 & 0.0129 & 0.0085 & $\begin{array}{l}0.0073 \\
\text { DTI75 }\end{array}$ & 0.0063 & 0.0055 & 0.0050 & 0.0047 & 0.0045 & 0.0042 \\
\hline & & & & & DTLZ5 & & & & & & \\
\hline & $\begin{array}{l}10 \\
20\end{array}$ & $\begin{array}{l}0.1407 \\
0.1571\end{array}$ & $\begin{array}{l}0.0068 \\
0.0096\end{array}$ & $\begin{array}{l}0.0057 \\
0.0069\end{array}$ & $\begin{array}{l}0.0054 \\
0.0063\end{array}$ & $\begin{array}{l}0.0050 \\
0.0056\end{array}$ & $\begin{array}{l}0.0046 \\
0.0053\end{array}$ & $\begin{array}{l}0.0042 \\
0.0052\end{array}$ & $\begin{array}{l}0.0042 \\
0.0050\end{array}$ & $\begin{array}{l}0.0042 \\
0.0049\end{array}$ & $\begin{array}{l}0.0041 \\
0.0048\end{array}$ \\
\hline K-RVEA & 30 & 0.1207 & 0.0111 & & $\begin{array}{l}0.0068 \\
0.0068\end{array}$ & 0.0063 & 0.0058 & $\begin{array}{l}0.0056 \\
0.0056\end{array}$ & $\begin{array}{l}0.0054 \\
0.0050\end{array}$ & $\begin{array}{l}0.0051 \\
0.0051\end{array}$ & $\begin{array}{l}0.0051 \\
0.0048\end{array}$ \\
\hline & 50 & 0.1584 & 0.0052 & 0.0046 & 0.0042 & 0.0041 & 0.0041 & 0.0040 & 0.0040 & 0.0039 & 0.0038 \\
\hline & 70 & 0.1368 & 0.0101 & 0.0072 & 0.0065 & 0.0062 & 0.0056 & 0.0055 & 0.0052 & 0.0050 & 0.0049 \\
\hline M-K-RVEA & $\mathrm{ADC}$ & 0.1286 & 0.0071 & 0.0055 & $\begin{array}{l}0.0048 \\
D T 56\end{array}$ & 0.0045 & 0.0042 & 0.0039 & 0.0039 & 0.0037 & 0.0037 \\
\hline & & & & & & & & & & & \\
\hline & $\begin{array}{l}10 \\
20\end{array}$ & $\begin{array}{l}0.9746 \\
0.9513\end{array}$ & $\begin{array}{l}0.4591 \\
0.4647\end{array}$ & $\begin{array}{l}\begin{array}{l}0.4409 \\
0.4897\end{array} \\
0\end{array}$ & $\begin{array}{l}\begin{array}{l}0.3822 \\
0.4926\end{array} \\
0.04\end{array}$ & 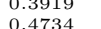 & $\begin{array}{l}\begin{array}{l}0.3913 \\
0.4403\end{array} \\
0\end{array}$ & $\begin{array}{l}0.3306 \\
0.4151\end{array}$ & $\begin{array}{l}0.3261 \\
0.3610\end{array}$ & $\begin{array}{l}0.3428 \\
0.3625\end{array}$ & $\begin{array}{l}\begin{array}{l}0.3140 \\
0.3456\end{array} \\
0.04\end{array}$ \\
\hline K-RVEA & 30 & 0.996 & 0.45 & & 0.4227 & 0.3993 & 0.3845 & & 0.0057 & 0.3867 & 年 \\
\hline & 50 & 0.9480 & 0.5927 & 0.5124 & 0.4898 & 0.4478 & 0.4311 & 0.4444 & 0.4308 & 0.4108 & 0.3957 \\
\hline & 70 & 0.9599 & 0.5421 & 0.4830 & 0.4446 & 0.4565 & 0.4716 & 0.4370 & 0.4339 & 0.4248 & 0.4184 \\
\hline M-K-RVEA & $\mathrm{ADC}$ & 1.0474 & 0.4951 & 0.5234 & $\begin{array}{l}0.5070 \\
\text { DTI77 }\end{array}$ & 0.4695 & 0.4490 & 0.4279 & 0.4099 & 0.3823 & 0.3597 \\
\hline & & & & & & & & & & & \\
\hline & $\begin{array}{l}10 \\
20\end{array}$ & $\begin{array}{l}2.0241 \\
2.6543\end{array}$ & $\begin{array}{l}0.0026 \\
0.0012\end{array}$ & $\begin{array}{l}0.0012 \\
0.0007\end{array}$ & $\begin{array}{l}0.0008 \\
0.0005\end{array}$ & $\begin{array}{l}0.0006 \\
0.0004\end{array}$ & $\begin{array}{l}0.0005 \\
0.0003\end{array}$ & $\begin{array}{l}0.0005 \\
0.0003\end{array}$ & $\begin{array}{l}0.0004 \\
0.0003\end{array}$ & $\begin{array}{l}0.0004 \\
0.0002\end{array}$ & $\begin{array}{l}0.0003 \\
0.0002\end{array}$ \\
\hline K-RVEA & 30 & $\begin{array}{l}2.6040 \\
2.6896\end{array}$ & 0.0009 & & & 0.0003 & & & & & \\
\hline & 50 & 2.3290 & 0.0010 & 0.0007 & 0.0005 & 0.0004 & 0.0004 & 0.0003 & 0.0003 & 0.0002 & $\begin{array}{l}2.0384 \\
2.0384\end{array}$ \\
\hline & 70 & 2.0943 & 0.0007 & 0.0005 & 0.0005 & 0.0004 & 0.0003 & 0.0003 & 0.0003 & 0.0002 & 0.0002 \\
\hline M-K-RVEA & $\mathrm{ADC}$ & 2.0241 & 0.0009 & 0.0007 & $\begin{array}{l}0.0004 \\
\text { DTI78 }\end{array}$ & 0.0004 & 0.0003 & 0.0003 & 0.0003 & 0.0002 & 0.0002 \\
\hline & & & & & & & & & & & \\
\hline & $\begin{array}{l}10 \\
20\end{array}$ & $\begin{array}{l}0.0548 \\
0.0587\end{array}$ & $\begin{array}{l}0.0322 \\
0.0327\end{array}$ & $\begin{array}{l}0.0322 \\
0.0327\end{array}$ & $\begin{array}{l}0.0322 \\
0.0327\end{array}$ & $\begin{array}{l}0.0322 \\
0.0327\end{array}$ & $\begin{array}{l}0.0322 \\
0.0327\end{array}$ & $\begin{array}{l}0.0322 \\
0.0327\end{array}$ & $\begin{array}{l}0.0322 \\
0.0327\end{array}$ & $\begin{array}{l}0.0322 \\
0.0327\end{array}$ & $\begin{array}{l}0.0322 \\
0.0327\end{array}$ \\
\hline K-RVEA & 30 & & & & & & & & & & \\
\hline & 50 & 0.072 & 0.0303 & 0.0303 & 0.0303 & 0.0303 & 0.0303 & 0.0303 & 0.0303 & 0.0303 & $\begin{array}{l}0.0303 \\
0.0303\end{array}$ \\
\hline & 70 & 0.0635 & 0.0357 & 0.0357 & 0.0357 & 0.0357 & 0.0357 & 0.0357 & 0.0357 & 0.0357 & 0.0357 \\
\hline M-K-RVEA & $\mathrm{ADC}$ & 0.0635 & 0.0310 & 0.0310 & 0.0310 & 0.0310 & 0.0310 & 0.0310 & 0.0310 & 0.0310 & 0.0310 \\
\hline & & & & & & & & & & & \\
\hline & $\begin{array}{l}10 \\
20\end{array}$ & $\begin{array}{l}3.4231 \\
6.3959 \\
\end{array}$ & $\begin{array}{l}2.2998 \\
2.5242\end{array}$ & $\begin{array}{l}1.5890 \\
2.3244\end{array}$ & 1.34 & $\begin{array}{l}1.4657 \\
1.9384\end{array}$ & 2.27 & $\begin{array}{l}1.4092 \\
2.3200\end{array}$ & 1.2228 & 1.1880 & $\begin{array}{l}1.1553 \\
2.1090\end{array}$ \\
\hline K-RVEA & 30 & & & & & & & & & & \\
\hline & 50 & 3.9907 & 1.5387 & 1.1807 & 0.9758 & 2.3 & 2.18 & $\begin{array}{l}1.8939 \\
1.839\end{array}$ & $\begin{array}{l}1.8939 \\
\end{array}$ & 1.8430 & 1.7425 \\
\hline & 70 & 4.5920 & 2.5457 & 1.9841 & 2.2016 & & & & & & 1.7881 \\
\hline M-K-RVEA & $\mathrm{ADC}$ & 3.7733 & 2.5289 & 2.2851 & 2.1059 & 2.0260 & 2.0260 & 1.8907 & 1.8280 & 1.2761 & 1.1746 \\
\hline
\end{tabular}


Table 3 IGD values of K-RVEA and M-K-RVEA on DTLZs benchmark set

\begin{tabular}{|c|c|c|c|c|c|c|c|c|c|c|c|}
\hline MOEAs & Evals & 2000 & 4000 & 6000 & 8000 & 10000 & 12000 & 14000 & 16000 & 18000 & 20000 \\
\hline K-RVEA & $\begin{array}{l}10 \\
20 \\
30 \\
50 \\
70 \\
\text { ADC }\end{array}$ & $\begin{array}{l}37.5017 \\
69.2926 \\
73.5400 \\
75.3314 \\
56.6293 \\
30.8156\end{array}$ & $\begin{array}{l}15.9551 \\
12.2018 \\
18.0276 \\
21.8503 \\
17.9351 \\
9.9032\end{array}$ & $\begin{array}{l}15.9551 \\
9.0805 \\
18.0276 \\
21.8503 \\
17.9351 \\
9.9032\end{array}$ & $\begin{array}{l}\text { DTLZ1 } \\
15.9551 \\
6.0770 \\
18.0276 \\
21.8503 \\
17.9351 \\
9.9032\end{array}$ & $\begin{array}{l}8.6306 \\
6.0770 \\
18.0276 \\
18.8011 \\
15.7866 \\
9.9032\end{array}$ & $\begin{array}{l}8.6306 \\
6.0770 \\
18.0276 \\
18.8011 \\
15.7866 \\
9.9032\end{array}$ & $\begin{array}{l}8.6306 \\
6.0770 \\
18.0276 \\
18.8011 \\
11.9698 \\
9.9032\end{array}$ & $\begin{array}{l}6.4437 \\
66.0770 \\
18.0276 \\
18.8011 \\
11.9998 \\
0.9028\end{array}$ & $\begin{array}{l}6.4437 \\
6.0770 \\
18.0276 \\
18.8011 \\
11.9698 \\
0.9032\end{array}$ & $\begin{array}{l}6.4437 \\
6.0770 \\
18.0276 \\
18.8011 \\
11.9698 \\
\end{array}$ \\
\hline M-K-RVEA & ADC & 30.8156 & 9.9032 & 9.9032 & $\begin{array}{l}9.9032 \\
\text { DTLZ2 }\end{array}$ & 9.9032 & 9.9032 & 9.9032 & 9.9032 & 9.9032 & 9.9032 \\
\hline K-RVEA & $\begin{array}{l}10 \\
20 \\
30 \\
50 \\
70 \\
\text { ADC }\end{array}$ & $\begin{array}{l}0.4863 \\
0.4862 \\
0.4720 \\
0.5233 \\
0.4848 \\
0.5617\end{array}$ & $\begin{array}{l}0.0523 \\
0.0561 \\
0.0467 \\
0.0476 \\
0.0502 \\
0.0528\end{array}$ & $\begin{array}{l}0.0417 \\
0.0368 \\
0.0382 \\
0.0387 \\
0.0427 \\
0.0398\end{array}$ & $\begin{array}{l}0.0372 \\
0.0325 \\
0.0326 \\
0.0340 \\
0.0372 \\
0.0354\end{array}$ & $\begin{array}{l}0.0343 \\
0.0297 \\
0.0293 \\
0.0306 \\
0.0338 \\
0.0335\end{array}$ & $\begin{array}{l}0.0326 \\
0.0287 \\
0.0283 \\
0.0295 \\
0.0324 \\
0.0320\end{array}$ & $\begin{array}{l}0.0314 \\
0.0281 \\
0.0268 \\
0.0285 \\
0.0302 \\
0.0305\end{array}$ & $\begin{array}{l}0.0302 \\
0.0273 \\
0.0260 \\
0.0276 \\
0.0292 \\
0.0205\end{array}$ & $\begin{array}{l}0.0293 \\
0.0265 \\
0.0249 \\
0.0272 \\
0.0283 \\
0.0287\end{array}$ & $\begin{array}{l}0.0284 \\
0.0259 \\
0.0244 \\
0.0267 \\
0.0272 \\
\end{array}$ \\
\hline M-K-RVEA & ADC & 0.5617 & 0.0528 & 0.0398 & $\frac{0.0354}{\text { DTLZ3 }}$ & 0.0335 & 0.0320 & 0.0305 & 0.0295 & 0.0287 & 0.0278 \\
\hline M-K-RVEA & $\begin{array}{l}10 \\
20 \\
30 \\
50 \\
70 \\
\text { ADC } \\
\end{array}$ & $\begin{array}{l}500.9795 \\
608.2876 \\
421.7119 \\
533.7593 \\
672.9475 \\
500.9795\end{array}$ & $\begin{array}{l}154.0806 \\
195.9133 \\
282.9219 \\
229.4352 \\
281.1927 \\
235.2239\end{array}$ & $\begin{array}{l}154.0806 \\
185.9003 \\
229.2645 \\
225.6308 \\
274.5212 \\
204.4000\end{array}$ & $\begin{array}{c}\text { DTLZ3 } \\
154.0806 \\
185.9003 \\
229.2645 \\
225.6308 \\
246.6838 \\
204.4000\end{array}$ & $\begin{array}{l}154.0806 \\
185.9003 \\
229.2645 \\
196.0629 \\
246.6838 \\
204.4000\end{array}$ & $\begin{array}{l}154.0806 \\
185.9003 \\
222.9678 \\
196.0629 \\
246.6838 \\
182.6566\end{array}$ & $\begin{array}{l}154.0806 \\
185.9003 \\
132.3652 \\
196.0629 \\
183.5229 \\
182.6566\end{array}$ & $\begin{array}{l}154.0806 \\
185.9003 \\
132.3652 \\
196.0629 \\
183.5229 \\
182.6566\end{array}$ & $\begin{array}{l}154.0806 \\
177.8553 \\
132.3652 \\
196.0629 \\
183.5229 \\
182.6566\end{array}$ & $\begin{array}{l}154.0806 \\
177.8553 \\
132.3652 \\
195.8602 \\
183.5229 \\
182.6566\end{array}$ \\
\hline NI-K-KVEA & ADC & 500.9795 & 235.2239 & 204.4000 & $\frac{204.4000}{\text { DTLZ4 }}$ & 204.4000 & 182.6566 & 182.6566 & 182.6566 & 182.6566 & 182.6566 \\
\hline K-RVEA & $\begin{array}{l}10 \\
20 \\
30 \\
50 \\
70 \\
\text { ADC } \\
\end{array}$ & $\begin{array}{l}0.9201 \\
0.9733 \\
0.8311 \\
0.8441 \\
0.8792 \\
0.8792 \\
\end{array}$ & $\begin{array}{l}0.1337 \\
0.0753 \\
0.0745 \\
0.0786 \\
0.0833 \\
0.0833\end{array}$ & $\begin{array}{l}0.0794 \\
0.0606 \\
0.0626 \\
0.0637 \\
0.0662 \\
0.0662\end{array}$ & $\begin{array}{l}0.0595 \\
0.0543 \\
0.0568 \\
0.0577 \\
0.0629 \\
0.0629\end{array}$ & $\begin{array}{l}0.0549 \\
0.0503 \\
0.0520 \\
0.0541 \\
0.0582 \\
0.0582\end{array}$ & $\begin{array}{l}0.0495 \\
0.0465 \\
0.0466 \\
0.0512 \\
0.0552 \\
0.0552\end{array}$ & $\begin{array}{l}0.0477 \\
0.0447 \\
0.0441 \\
0.0474 \\
0.0542 \\
0.0542\end{array}$ & $\begin{array}{l}0.0458 \\
0.0418 \\
0.0421 \\
0.0442 \\
0.0524 \\
0.0524\end{array}$ & $\begin{array}{l}0.0444 \\
0.0408 \\
0.0408 \\
0.0420 \\
0.0512 \\
0.0512\end{array}$ & $\begin{array}{l}0.0434 \\
0.0396 \\
0.0399 \\
0.0413 \\
0.0504 \\
0.0504\end{array}$ \\
\hline & & 0.8192 & 0.0833 & & DTLZ5 & & & & & & \\
\hline K-RVEA & $\begin{array}{l}10 \\
20 \\
30 \\
50 \\
70 \\
\end{array}$ & $\begin{array}{l}0.3699 \\
0.3739 \\
0.4081 \\
0.3483 \\
0.3860 \\
\end{array}$ & $\begin{array}{l}0.0395 \\
0.0583 \\
0.0722 \\
0.0381 \\
0.0494 \\
\end{array}$ & $\begin{array}{l}0.0397 \\
0.0525 \\
0.0597 \\
0.0337 \\
0.0426 \\
\end{array}$ & $\begin{array}{l}0.0384 \\
0.0391 \\
0.0496 \\
0.0317 \\
0.0412 \\
\end{array}$ & $\begin{array}{l}0.0343 \\
0.0371 \\
0.0492 \\
0.0307 \\
0.0411 \\
\end{array}$ & $\begin{array}{l}0.0330 \\
0.0357 \\
0.0478 \\
0.0367 \\
0.0394 \\
\end{array}$ & $\begin{array}{l}0.0329 \\
0.0355 \\
0.0457 \\
0.0364 \\
0.0393 \\
\end{array}$ & $\begin{array}{l}0.0327 \\
0.0347 \\
0.0449 \\
0.0364 \\
0.0388 \\
\end{array}$ & $\begin{array}{l}0.0301 \\
0.0345 \\
0.0421 \\
0.0364 \\
0.0384 \\
\end{array}$ & $\begin{array}{l}0.0301 \\
0.0344 \\
0.0421 \\
0.0363 \\
0.0376 \\
\end{array}$ \\
\hline M-K-RVEA & ADC & 0.4247 & 0.0421 & 0.0371 & $\frac{0.0380}{0}$ & 0.0373 & 0.0345 & 0.0338 & 0.0336 & 0.0333 & 0.0333 \\
\hline K-RVEA & $\begin{array}{l}10 \\
20 \\
30 \\
50 \\
70\end{array}$ & $\begin{array}{l}8.2380 \\
8.4386 \\
8.2757 \\
8.2283 \\
8.4906\end{array}$ & $\begin{array}{l}1.8545 \\
2.2229 \\
2.4312 \\
2.7022 \\
2.4451\end{array}$ & $\begin{array}{l}0.8540 \\
1.3579 \\
2.4304 \\
2.5015 \\
2.4451\end{array}$ & $\begin{array}{l}0.8110 \\
1.3532 \\
1.8914 \\
2.4640 \\
2.4451\end{array}$ & $\begin{array}{l}0.8110 \\
1.3532 \\
1.7946 \\
2.4640 \\
2.1171\end{array}$ & $\begin{array}{l}0.8110 \\
1.2986 \\
1.7637 \\
2.4640 \\
2.0384\end{array}$ & $\begin{array}{l}0.8110 \\
1.2986 \\
1.6556 \\
2.2781 \\
2.0384\end{array}$ & $\begin{array}{l}0.8110 \\
1.2557 \\
1.6556 \\
2.2781 \\
2.0384\end{array}$ & $\begin{array}{l}0.7906 \\
1.2557 \\
1.6556 \\
2.0575 \\
2.0384\end{array}$ & $\begin{array}{l}0.7724 \\
1.2557 \\
1.6437 \\
2.0575 \\
2.0384\end{array}$ \\
\hline M-K-RVEA & $\mathrm{ADC}$ & 8.4051 & 2.9249 & 1.8047 & 1.6488 & 1.6488 & 1.6488 & 1.6488 & 1.6334 & 1.5731 & 1.5731 \\
\hline K-RVEA & $\begin{array}{l}10 \\
20 \\
30 \\
50 \\
70 \\
\end{array}$ & $\begin{array}{l}8.1067 \\
7.4668 \\
8.6059 \\
8.8847 \\
8.2937 \\
8\end{array}$ & $\begin{array}{l}0.0611 \\
0.0492 \\
0.0514 \\
0.0707 \\
0.0725\end{array}$ & $\begin{array}{l}0.0397 \\
0.0406 \\
0.0424 \\
0.0599 \\
0.0598\end{array}$ & $\begin{array}{l}\text { DTLZ } \\
0.0332 \\
0.0338 \\
0.0380 \\
0.0543 \\
0.0559 \\
\end{array}$ & $\begin{array}{l}0.0295 \\
0.0317 \\
0.0358 \\
0.0469 \\
0.0506\end{array}$ & $\begin{array}{l}0.0276 \\
0.0296 \\
0.0341 \\
0.0440 \\
0.0487\end{array}$ & $\begin{array}{l}0.0256 \\
0.0280 \\
0.0312 \\
0.0420 \\
0.0459\end{array}$ & $\begin{array}{l}0.0243 \\
0.0268 \\
0.0303 \\
0.0407 \\
0.0442\end{array}$ & $\begin{array}{l}0.0231 \\
0.0256 \\
0.0293 \\
0.0398 \\
0.0433\end{array}$ & $\begin{array}{l}0.0222 \\
0.0246 \\
0.0282 \\
0.0477 \\
0.0424\end{array}$ \\
\hline M-K-RVEA & $\mathrm{ADC}$ & 8.1067 & 0.0618 & 0.0546 & 0.0456 & 0.0433 & 0.0411 & 0.0359 & 0.0343 & 0.0320 & 0.0312 \\
\hline K-RVEA & $\begin{array}{l}10 \\
20 \\
30 \\
50 \\
70 \\
\end{array}$ & $\begin{array}{l}0.2309 \\
0.2590 \\
0.2190 \\
0.2516 \\
0.2397\end{array}$ & $\begin{array}{l}0.2050 \\
0.1599 \\
0.1827 \\
0.2357 \\
0.1935\end{array}$ & $\begin{array}{l}0.2050 \\
0.1599 \\
0.1827 \\
0.2357 \\
0.1935\end{array}$ & $\begin{array}{c}\text { DTLZ } \\
0.2050 \\
0.1599 \\
0.1827 \\
0.2357 \\
0.1935\end{array}$ & $\begin{array}{l}0.2050 \\
0.1599 \\
0.1827 \\
0.2357 \\
0.1935\end{array}$ & $\begin{array}{l}0.2050 \\
0.1599 \\
0.1827 \\
0.2357 \\
0.1935\end{array}$ & $\begin{array}{l}0.2050 \\
0.1599 \\
0.1827 \\
0.2357 \\
0.1935\end{array}$ & $\begin{array}{l}0.2050 \\
0.1599 \\
0.1827 \\
0.2357 \\
0.1935\end{array}$ & $\begin{array}{l}0.2050 \\
0.1599 \\
0.1827 \\
0.2357 \\
0.1935\end{array}$ & $\begin{array}{l}0.2050 \\
0.1599 \\
0.1827 \\
0.2357 \\
0.1935\end{array}$ \\
\hline M-K-RVEA & $\mathrm{ADC}$ & 0.2397 & 0.1790 & 0.1790 & 0.1790 & 0.1790 & 0.1790 & 0.1790 & 0.1790 & 0.1790 & 0.1790 \\
\hline K-RVEA & $\begin{array}{l}10 \\
20 \\
30 \\
50 \\
70 \\
\end{array}$ & $\begin{array}{l}11.3847 \\
11.1618 \\
11.1510 \\
11.2579 \\
11.1964 \\
\end{array}$ & $\begin{array}{l}6.3491 \\
6.3076 \\
7.7752 \\
7.9947 \\
6.6274 \\
\end{array}$ & $\begin{array}{l}6.1299 \\
6.2910 \\
7.7752 \\
7.9363 \\
6.3928 \\
\end{array}$ & $\begin{array}{l}\text { DTLZ } \\
6.1299 \\
6.2910 \\
7.7563 \\
7.9294 \\
6.3837 \\
\end{array}$ & $\begin{array}{l}5.6413 \\
6.2910 \\
7.7378 \\
7.5453 \\
6.3837 \\
\end{array}$ & $\begin{array}{l}5.3711 \\
6.2910 \\
7.7212 \\
7.5453 \\
6.3837 \\
\end{array}$ & 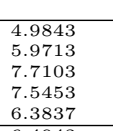 & $\begin{array}{l}.9843 \\
5.9713 \\
7.7103 \\
7.5453 \\
6.3837 \\
\end{array}$ & $\begin{array}{l}4.9843 \\
5.9713 \\
7.7103 \\
7.5453 \\
6.3837 \\
\end{array}$ & $\begin{array}{l}4.9843 \\
5.9066 \\
7.7099 \\
7.5453 \\
6.3830 \\
\end{array}$ \\
\hline M-K-RVEA & ADC & 11.2529 & 6.5146 & 6.4943 & 6.4943 & 6.4943 & 6.4943 & 6.4943 & 6.4943 & 6.4697 & 6.4697 \\
\hline
\end{tabular}




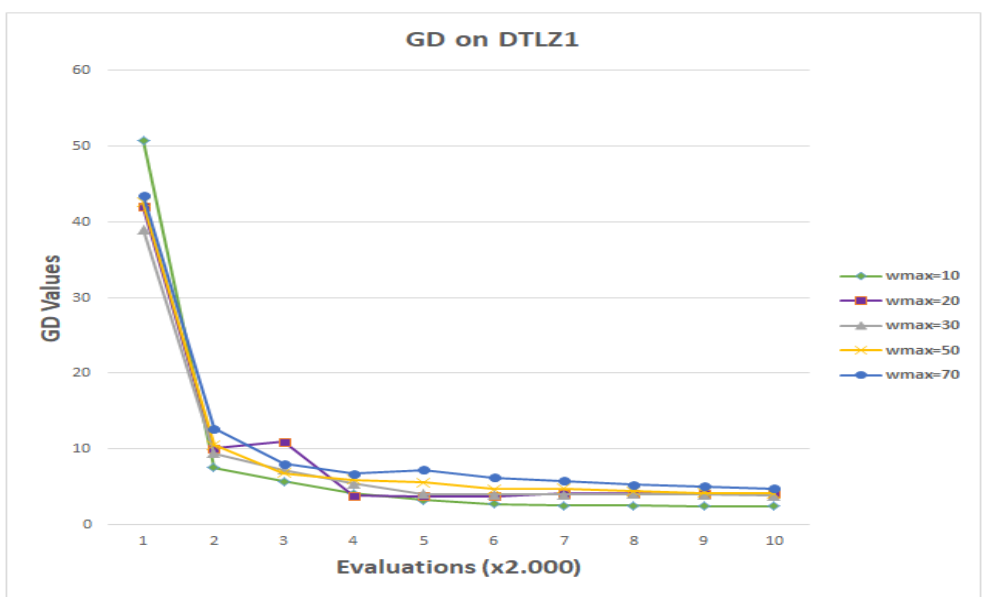

Fig. 6 The behavior of K-RVEA on DTLZ1 (GD)

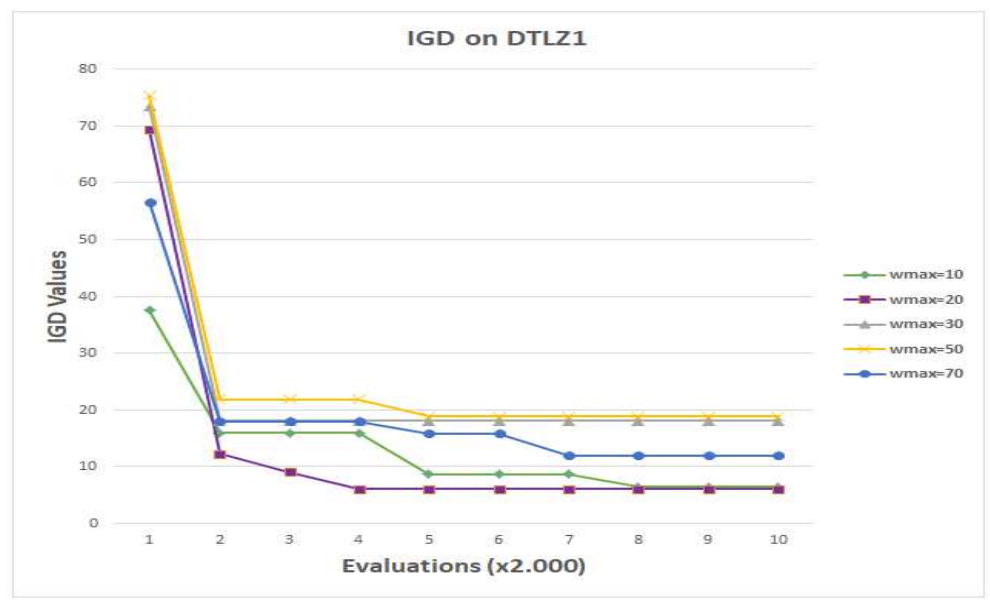

Fig. 7 The behavior of K-RVEA on DTLZ1 (IGD)

CSEA: Selecting a reference solution is an important factor for the performance of the algorithm. The space division is based on a boundary that is formed from the selected solutions. A key parameter for CSEA is $K$ : number of reference solutions that are selected. The role of $K$ is discussed in the proposal. When the value of $K$ is small, the number of reference solutions of category II solutions is small. The algorithm has better convergence performance but is poor in terms of diversity. In contrast, a larger value for $K$ will produce better diversity but poorly convergence. The choice of the number of reference solutions provides a way of balancing the convergence and diversity of the selected category II solutions. 


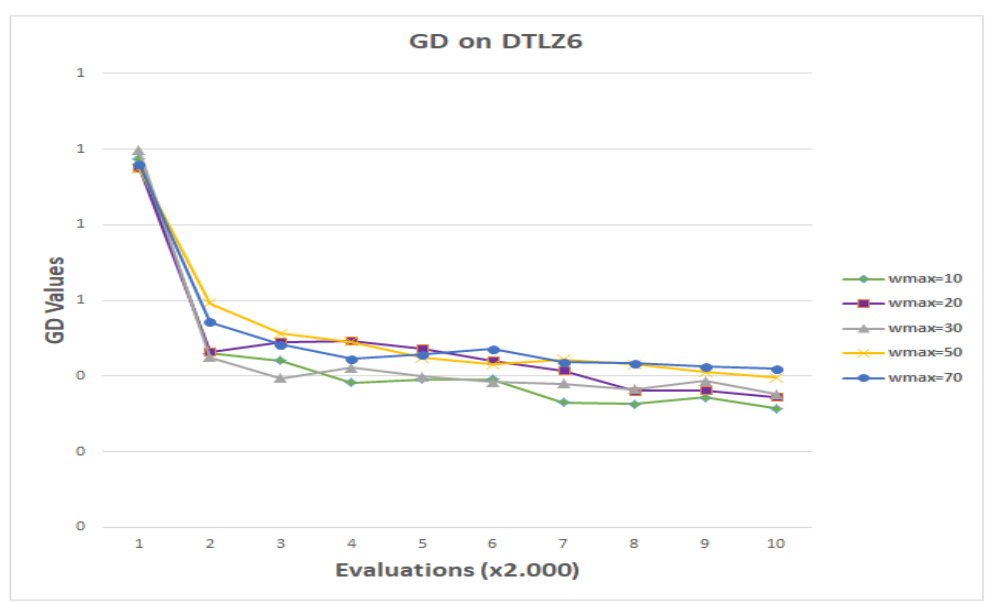

Fig. 8 The behavior of K-RVEA on DTLZ6 (GD)

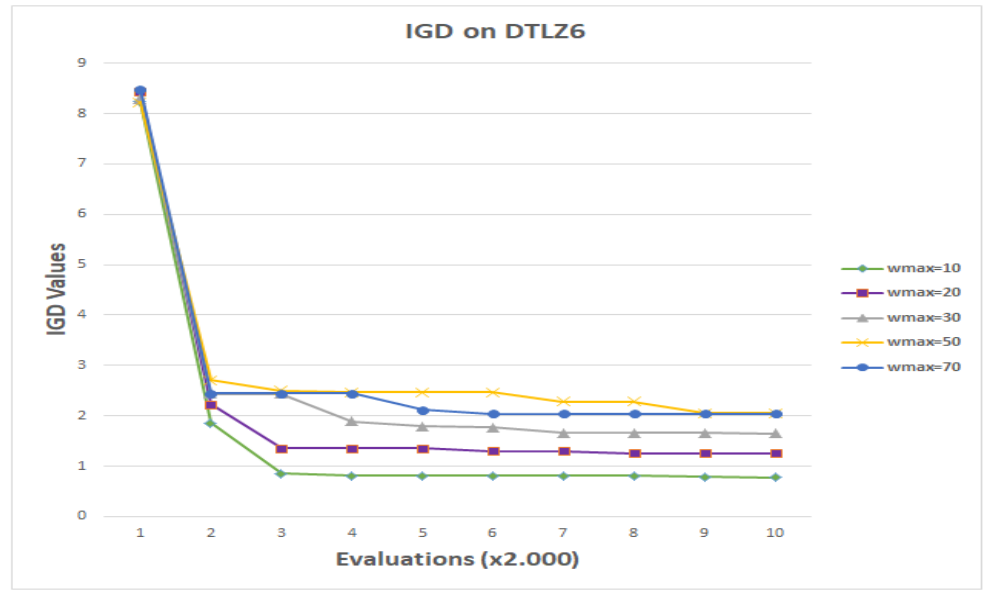

Fig. 9 The behavior of K-RVEA on DTLZ6 (IGD)

The authors empirically investigated the impact of the number of reference solutions $K$ on the performance of CSEA for solving problems with different numbers of objectives. CSEA with different parameter $K$ is tested on different benchmark sets. From the results, the best choice of $K$ is recorded to the algorithm. The parameter is then fixed at six for all benchmark sets. This is the practical parameter value. Similar to the analysis of the evolutionary process behavior in K-RVEA, the convergence in the early stages and the divergence of the population in the end-stage should be enhanced. CSEA uses $K$ parameter to select reference solutions to form the classification boundary, a radial space division from the selection strategy. Because $K$ is not adaptive, it lacks adaptability and makes noises, which is against the robustness of the algorithm. 
3.3 Guidance techniques

\subsubsection{An adaptive control}

From the experimental results with K-RVEA, for reducing the noise, appropriately adjusting parameters during evolution creates an effective balance between convergence and diversity of the population. Specifically, adjust the number of generations before updating the model according to the assessed factors and assume that the direct influence in SAEAs is the processing time of the evolution, quality of the current population, and computational characteristics of the problem.

The use of surrogate function and frequency of updating the Kriging model flexibly depends on the quality of the population in the current generation. To determine the quality of the solution, we use two defined values at a time $t$, with the test problem prb as follows.

$$
Q_{t}=\frac{n_{\text {non }}}{N_{P}} * \frac{F E}{F E_{\max }} * C_{p r b}
$$

where, $n_{n o n}$ is the number of non-dominated solutions in the main population, $N_{P}$ is the size of the population, $F E$ is the number of function evaluations, $F E_{\text {max }}$ is the maximum number of function evaluations. $C_{p r b}$ is the complexity of the problem, which depends on the complexity of the test function, size of decision spaces, number of objectives and number of calculations for objective functions. We choose practical values $C_{p r b} \in[0.8,1]$, the default value is one. The constant 60 is a generated point to control $w_{\max }$ in range $[10,50]$. From on the $Q_{t}$ value, we will calculate the value of $w_{\max } \in R$ (the rate of using surrogate function and the frequency of updating the Kriging model) in the range, a normalize function is defined to adjust valid $w_{\max }$ values.

$$
w_{\max }=\text { Normalize }\left(Q_{t} * 60\right)
$$

The modified version M-K-RVEA is described in Algorithm 1.

\subsubsection{An adaptive number of reference solutions}

Analyzing the results of CSEA, to reduce the noise by balancing the evolution between exploration and mining and to maintain the convergence and diversity of the population, the selection of a reference solution requires adaptability. It depends on factors that we can identify with the following influences:

- The process of evolution: evolution occurs from the initial state of creation, the central stage, and the final stage. The quality of the main population (number of non-dominated solutions) at each stage is different. Therefore, reference solutions should be adjusted according to the quality of the current population in terms of convergence and diversity. Thus, it is necessary to select several different reference solutions to create a balance between exploration and exploitation, convergence, and diversity, respectively. 


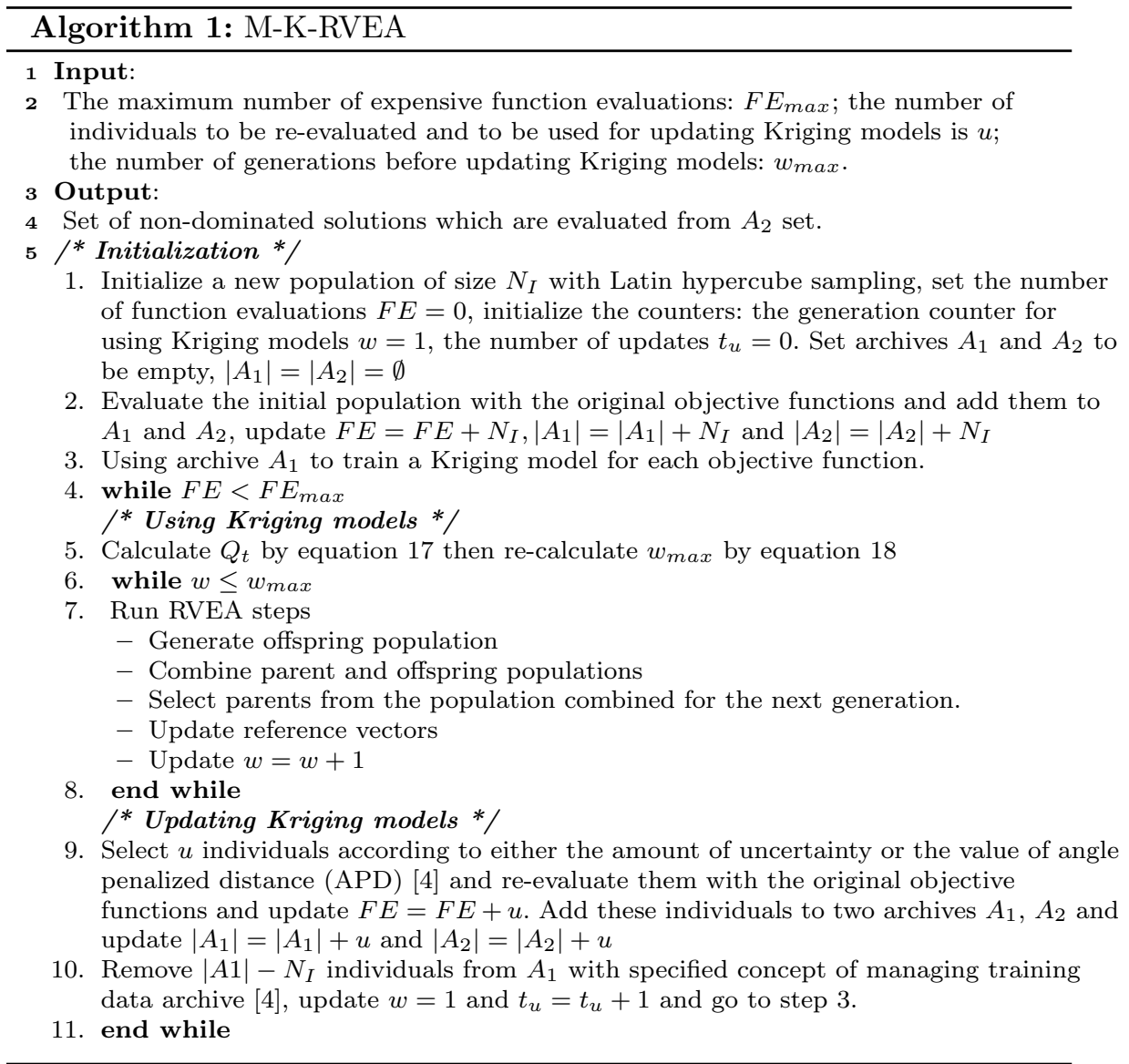

- The sample problems are different, the quality of the population in each stage has different development. At each time, based on the population, we choose the appropriate number of reference solutions. It makes the algorithm highly adaptive in maintaining the balanced convergence quality and diversity of the population.

- The difficulty of the problems is different. Making predictions on the problem, deciding on the reference solution from users is also an important factor to guide the evolution flexibly in maintaining the balance between exploration and mining. This helps create leaps that can overcome local points and the combination of decision-makers and evolution.

Based on the analysis from the experimental results above, appropriately adjusting parameters during evolution to create an effective balance between convergence quality and diversity of the population is necessary. Adjusting the number of generations before updating the model according to the assessed factors and assuming the direct influence in algorithms using surrogate 
function is the processing time of the evolution, the quality of the current population, and the computational characteristics of the problem.

Designing a dynamic selection strategy for selecting reference solutions to form the boundary for the classification considers the influence of the quality of the solution population in the current generation. Similar to the adaptive control technique for K-RVEA, to determine the quality of the solution. We calculate $Q_{t}$ value by using equation 17 . Based on the value of $Q_{t}$, we calculate the value of $K \in R$ (the number of reference solutions that will be selected to form the boundary) in the range $[3,9]$. A normalized function is defined to adjust valid $K$ values. We use six as a generated point to control the $K$ in the above range.

$$
K=\operatorname{Normalize}\left(Q_{t} * 6\right)
$$

The modified version M-CSEA is described in Algorithm 2.

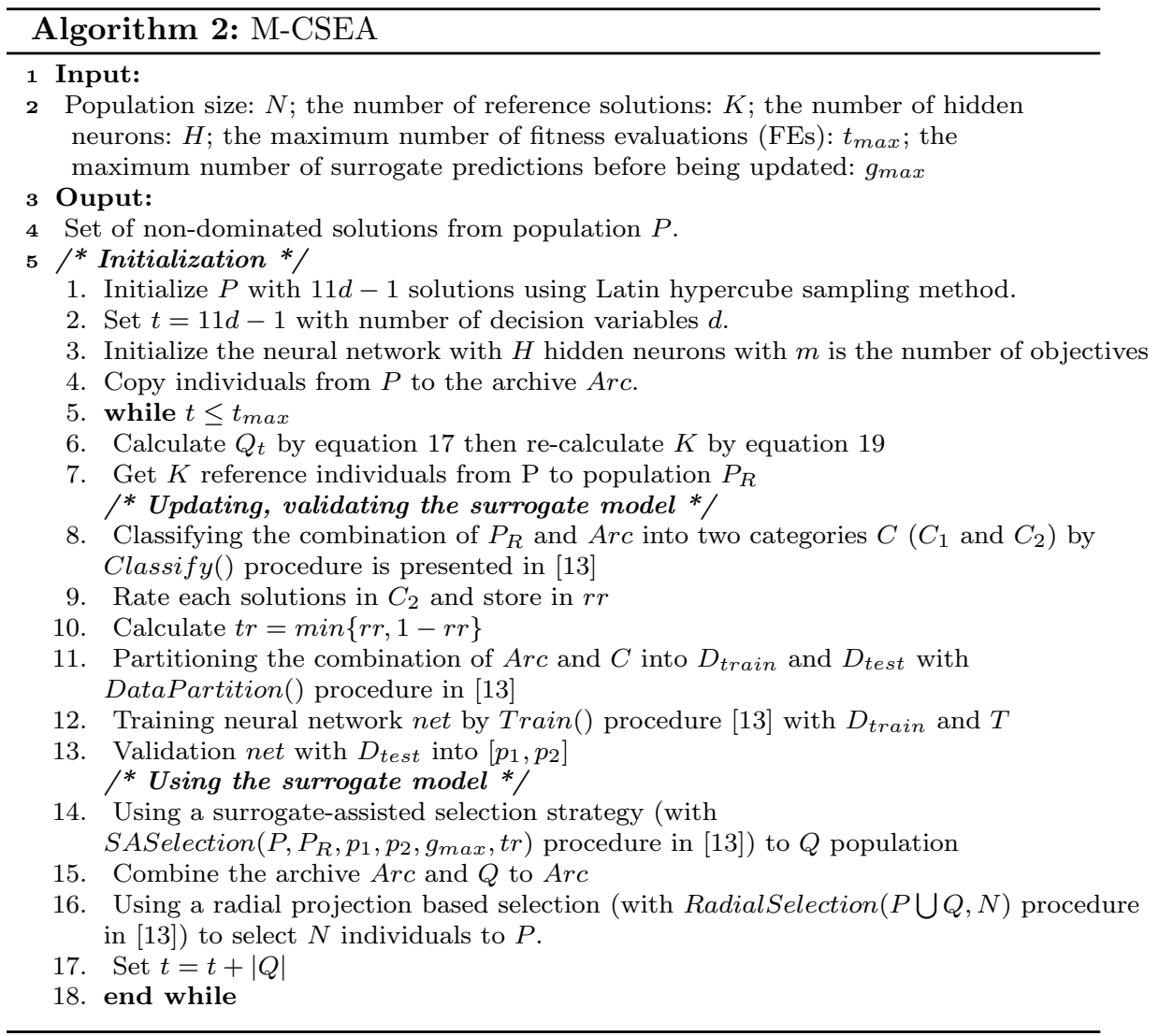




\subsection{Guidance experiments}

In the experiments, we used the K-RVEA and the modified M-K-RVEA with the above adaptive $w_{\max }$, and the CSEA and the modified M-CSEA with the dynamic K. We selected expensive problems in DTLZs [5] benchmark set. In the benchmark set, we have multiple kinds of expensive problems.

In our experimental parameters are the distribution index of crossover $n_{c}=20$ and the distribution index of mutation $n_{m}=20$, the crossover probability $p c=1.0$ and the mutation probability $p_{m}=1 / d$, where $d$ is the number of decision variables. For each case study, we run 30 independent times for analyzing experimental results.

Performance metrics are used to compare algorithms to form an understanding of which algorithm is better and in what aspects. However, it is difficult to define a concise algorithmic performance. When comparing, several criteria are employed [19]. Since each metric has both advantages and disadvantages, we will look at all two popular criteria: the GD and the inverse generational distance (IGD).

\subsection{The effectiveness of the guidance}

\subsubsection{M-K-RVEA}

To analyze the performance of M-K-RVEA, with a maximum of 20000 evaluations in 30 independent runs, we reported the results of GD, IGD measurement in rows ADC on Tables 2, 3. We also reported and compared the behaviors of K-RVEA (with the default $w_{\max }$ is 30 ) and M-K-RVEA.

From the result, M-K-RVEA with adaptive parameter control gets more balance of convergence and diversity for the obtained population. In the early stages, it gets better with 5 problems with GD (DTLZ1, DTLZ3, DTLZ4, DTLZ5, DTLZ8) and 6 problems on IGD (DTLZ1, DTLZ3, DTLZ4, DTLZ5, DTLZ8, DTLZ9). During the generations (the later stages), M-K-RVEA gets better in 5 problems on GD (DTLZ1, DTLZ3, DTLZ4, DTLZ5, DTLZ8). Results are better in 7 problems on IGD (DTLZ1, DTLZ3, DTLZ4, DTLZ5, DTLZ6, DTLZ8, DTLZ9). In the last stages, M-K-RVEA gets better in 6 problems on GD (DTLZ1, DTLZ3, DTLZ4, DTLZ5, DTLZ8, DTLZ9) and 6 problems in IGD (DTLZ1, DTLZ4, DTLZ5, DTLZ6, DTLZ8, DTLZ9). In other problems, K-RVEA gets the same results with M-K-RVEA and better than M-K-RVEA.

Looking at the final results obtained after 20000 evaluations, the results are basically better. However, with problems DTLZ2 and DTLZ7, M-KRVEA did not give improved results. With the feature that Pareto Front is located on the first quadrant of the unit sphere, the objective functions are the coordinate axes (then the ideal point is the origin), the results of two algorithms with DTLZ2 are quite similar (small error) on both GD and IGD measures. 
The correlation properties between the objective functions, and the geometric properties of Pareto Front with the coordinate axes are quite large, so the process of convergence (or achieving non-dominated solutions is increasing, uniformly), which can make the adaptive adjustment, not have much impact on the solution set search process.

With problem DTLZ7, with Pareto Front's features as disjoint regions, the convergence properties of the population obtained between two algorithms are similar (same GD value), however, evaluate both convergence factors and diversity (as measured by IGD), the maintenance of diversity of M-K-RVEA in this case is worse. In M-K-RVEA adjustment of the convergence rate, the multiplicative nature of M-K-RVEA does not take into account the exclusion of discontinuous regions that may be the cause of the proposed reduction in efficiency.

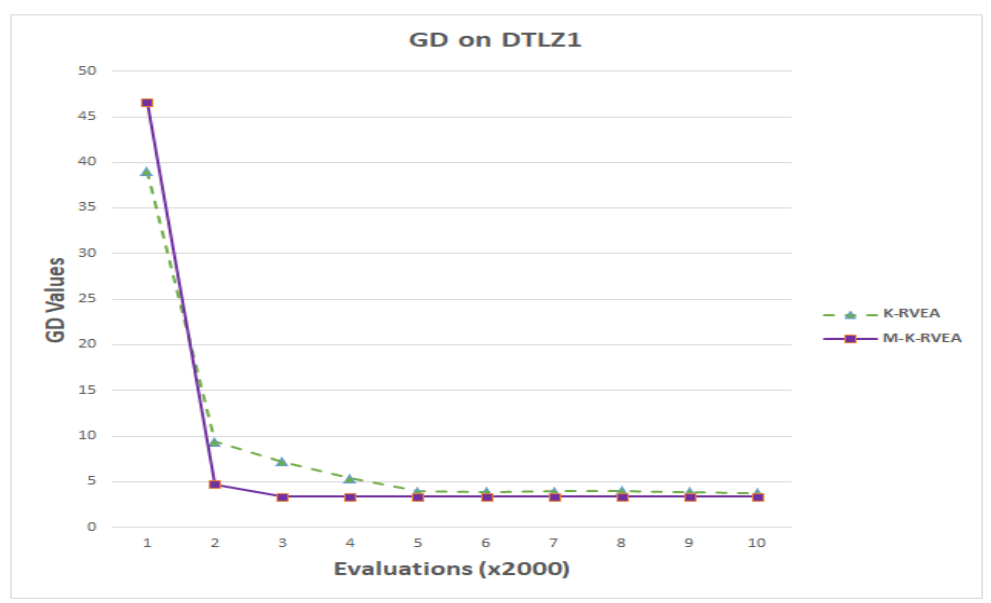

Fig. 10 The behavior of M-K-RVEA and K-RVEA on DTLZ1 (GD)

From the results of K-RVEA with the adaptive parameter control that affects implementing the optimal objective optimization algorithm using general surrogate functions, algorithms based on the Kriging model in particular. The use of the response parameter is to assess the quality of the population at the time of determining whether to update the Kriging model or not? The results using adaptive parameter control demonstrate the impact of the time, the current convergence quality of the population, and the characteristics of the problem on maintaining the equilibrium of convergence quality and diversity of the population, through ensuring a balance between exploration and exploitation process of evolution. The effectiveness of parameter selection in the current stages also depends on the quality and effectiveness of the previous stage, proving that a timely adjustment to maintain a balance between exploration and exploitation is needed. However, K-RVEA has better quality, although not large. It also gives us issues that need to be adequately addressed 


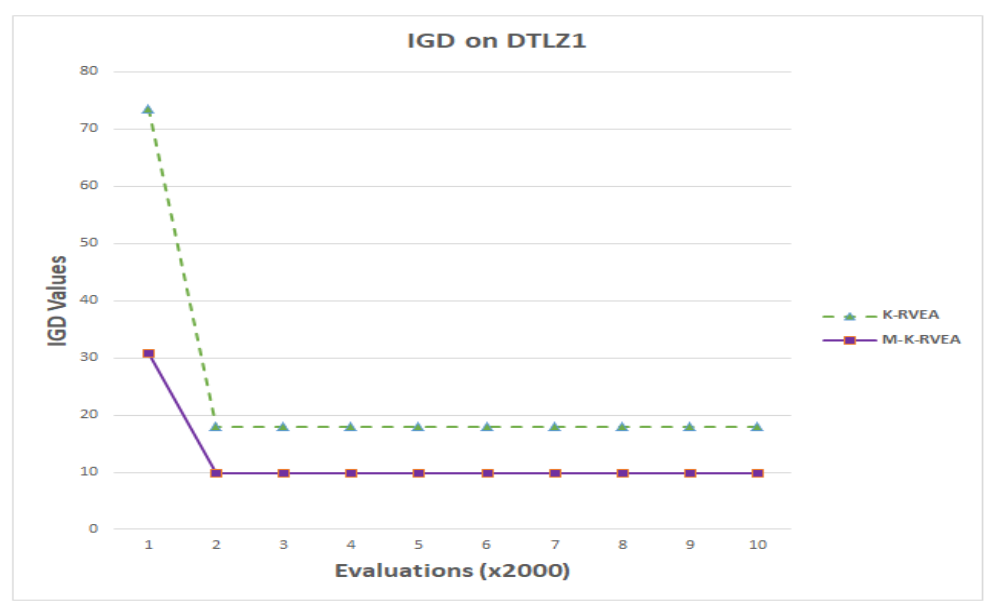

Fig. 11 The behavior of M-K-RVEA and K-RVEA on DTLZ1 (IGD)

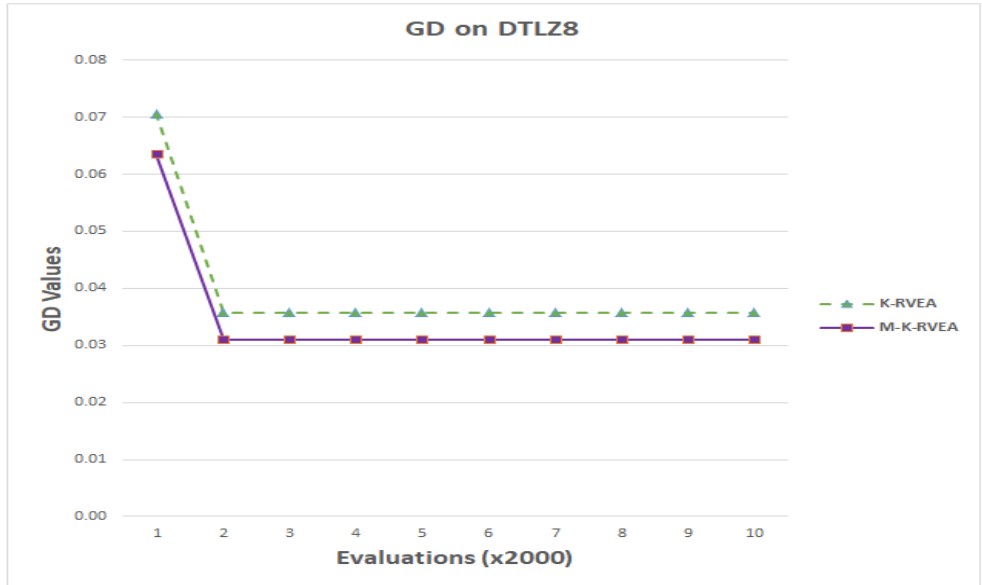

Fig. 12 The behavior of M-K-RVEA and K-RVEA on DTLZ8 (GD)

such as stability, sustainability, random factors course, etc. These factors influence the choice of parameters appropriately or propel evolution to better efficiency. It reduces noise to improve the robustness of the algorithm.

\subsubsection{M-CSEA}

The results of the M-CSEA are shown in in Table 4 and Table 5. We also reported and compared the behaviors of CSEA (with the default $K$ is 6 ) and M-CSEA, some of screenshots are shown in Figures: 14 to 17. 


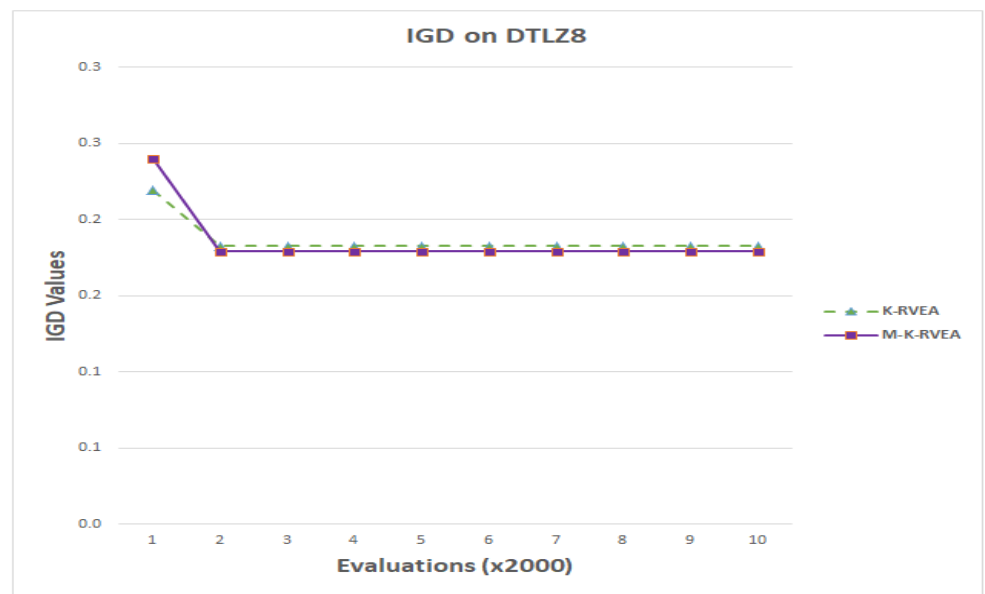

Fig. 13 The behavior of M-K-RVEA and K-RVEA on DTLZ8 (IGD) 
Table 4 GD values of CSEA and M-CSEA on DTLZs benchmark set

\begin{tabular}{|c|c|c|c|c|c|c|c|c|c|c|}
\hline MOEAs & 1000 & 2000 & 3000 & 4000 & 5000 & 6000 & 7000 & 8000 & 9000 & 10000 \\
\hline & & & & & $\begin{array}{r}\text { DTLZ1 } \\
0.049841399\end{array}$ & & & 678244 & & \\
\hline M-CSEA & 34.55195154 & 7.293731419 & $\begin{array}{r}0.093410808969 \\
1.659906579\end{array}$ & 0.0074109306 & 0.0498413597 & 0.0939185118 & 0.049742977 & 0.045665124 & $\begin{array}{c}0.038506013 \\
0.007362405\end{array}$ & $\begin{array}{c}0.023741603 \\
0.004428484\end{array}$ \\
\hline & & & & & DTLZ2 & & & & & \\
\hline$\frac{\text { CSEA }}{\text { M-CSEA }}$ & $\frac{0.111937965}{0.11952606}$ & $\begin{array}{l}0.003129364 \\
0.003268781\end{array}$ & $\begin{array}{l}0.001690321 \\
0.01166987\end{array}$ & $\begin{array}{l}0.001120471 \\
0.00817451\end{array}$ & $\frac{0.00073779}{0.000578712}$ & $\begin{array}{l}0.000533606 \\
0.000431733\end{array}$ & $\begin{array}{l}0.000432797 \\
0.00034105\end{array}$ & $\begin{array}{l}0.000362262 \\
0.000305767\end{array}$ & 0.000307509 & $\begin{array}{l}0.000255133 \\
0.000231731\end{array}$ \\
\hline & & & & & DTLZ3 & & & & & \\
\hline CSEA & 183.1624496 & 55.90315143 & $\begin{array}{l}19.85598041 \\
\end{array}$ & 14.28060864 & 11.19028972 & 8.806444816 & 6.527776933 & 5.115575763 & 3.5795702344 & 2.017174022 \\
\hline M-CSEA & & & & 9.138374728 & $\begin{array}{r}6.40377985 \\
\text { DTLZ4 }\end{array}$ & & & & & \\
\hline M-CSEA & 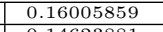 & 0.009324148 & & & 000863089 & & 0.00043422 & 0.000366466 & 0.000306355 & 0.000280448 \\
\hline M-CSEA & 0.14623881 & 0.00786399 & 0.002191418 & 0.001288786 & $\frac{000863088}{\text { DTLZ5 }}$ & 0.000676222 & & 0.000471852 & 0.000425611 & \\
\hline CSEA & 0.143045187 & 0.009094896 & 0.002914526 & 0.001666095 & 0.000700365 & 0.00038713 & 0.000316341 & 0.000294842 & 0.000244925 & 0.000211265 \\
\hline M-CSEA & 0.129459625 & 0.005469629 & 0.002643803 & 0.001694055 & 00970129 & 0.000418626 & 0.000343096 & 0.000282539 & 0.000252634 & \\
\hline & & & & & & & & & & \\
\hline M-CSEA & $\begin{array}{l}0.937372419 \\
0.982408347\end{array}$ & $\begin{array}{l}0.4992577544 \\
0.392787218\end{array}$ & $\frac{3.61595 \mathrm{E}-06}{0.103280342}$ & $\begin{array}{l}1.61995 \mathrm{E}-06 \\
0.001726882\end{array}$ & $\begin{array}{l}1.18003 \mathrm{E}-06 \\
0.000270277\end{array}$ & $\begin{array}{l}9.76567 \mathrm{E}-07 \\
0.000137908\end{array}$ & $\begin{array}{l}8.60556 \mathrm{E}-07 \\
1.7537 \mathrm{E}-05\end{array}$ & $\begin{array}{l}7.69527 \mathrm{E}-07 \\
1.31121 \mathrm{E}-05\end{array}$ & $\begin{array}{l}7.12257 \mathrm{E}-07 \\
7.19235 \mathrm{E}-06\end{array}$ & $\frac{6.60703 \mathrm{E}-07}{5.97902 \mathrm{E}-06}$ \\
\hline & & & & & DT & & & & & 3307 \\
\hline M-CSEA & $\frac{2.115099040}{2.631468079}$ & $\begin{array}{l}0.051293186 \\
0.051293186\end{array}$ & 0.0080460607 & 0.00309386 & \begin{tabular}{|l}
0.001154022 \\
0.00150
\end{tabular} & 0.000798814 & 0.0000458733 & $\begin{array}{l}.000401031 \\
0.000350707\end{array}$ & $\begin{array}{l}.000384502 \\
0.000289746\end{array}$ & 0.0000250762 \\
\hline & & & & & & & & & & \\
\hline CSEA & & & & & & & & & & \\
\hline M-CSEA & 0.052658985 & 0.015295372 & 0.015295372 & 0.015295372 & 0.015295372 & 0.015295372 & & 0.015295372 & 0.015295372 & 0.015295372 \\
\hline
\end{tabular}


Table 5 IGD values of CSEA and M-CSEA on DTLZs benchmark set

\begin{tabular}{|c|c|c|c|c|c|c|c|c|c|c|}
\hline MOEAs & 1000 & 2000 & 3000 & 4000 & 5000 & 6000 & 7000 & 8000 & 99000 & 10000 \\
\hline$\overline{C S F A}$ & 55.70363999 & 0.984059887 & 0.535546843 & 0.422683497 & $\begin{array}{c}\text { DTLZ1 } \\
0.319925917\end{array}$ & & & & & \\
\hline M-CSEA & $\begin{array}{l}05.1503999 \\
67.37843306\end{array}$ & $\begin{array}{l}0.984059886 \\
0.963184028 \\
\end{array}$ & $\frac{0.5350368445}{0.503859492}$ & $\begin{array}{l}0.42083496 \\
0.390551114\end{array}$ & $\frac{0.319935967}{0.29077677}$ & 0.549641266 & $\begin{array}{l}0.304424468 \\
0.238247688\end{array}$ & $\begin{array}{l}0.303095285 \\
0.230690889 \\
\end{array}$ & $\frac{0.25644543}{0.083235634}$ & $\frac{0.05960302}{0.05616082}$ \\
\hline & & & 0074753971 & & $\begin{array}{c}\text { DTLZ2 } \\
0.57251699\end{array}$ & & & & & \\
\hline M-CSEA & 0.445656061 & 0.105494378 & 0.077526559 & 0.069578 & 064227686 & 0.05 & 0.056 & & & \\
\hline & & & & & DTLZ3 & & & & & \\
\hline M-CSEA & 445.3088212 & $\begin{array}{l}137.0799636 \\
81.03428807\end{array}$ & 48.27255007 & 34.01990624 & $\begin{array}{l}51.0949479 \\
19.12160089\end{array}$ & $\begin{array}{r}32.67368451 \\
9.809608997\end{array}$ & $\frac{24.24581574}{8.919420738}$ & $\frac{15.80437507}{3.96288272}$ & $\frac{10.63840422}{3.06367452}$ & $\frac{9.8721718}{2.820373235}$ \\
\hline & & & & & DTLZ4 & & & & & \\
\hline M-CSEA & $\begin{array}{l}0.920595015 \\
0.867610961 \\
\end{array}$ & 0.17499921 & $\begin{array}{l}0.08340208 \\
0.074209237\end{array}$ & $\begin{array}{l}0.05060319 \\
0.05987287\end{array}$ & $\begin{array}{l}0.0651877807 \\
0.052081465\end{array}$ & $\begin{array}{l}0.056606665 \\
0.048150374\end{array}$ & $\begin{array}{l}0.059552637 \\
0.044544918\end{array}$ & \begin{tabular}{|l}
0.051886509 \\
0.041799879
\end{tabular} & $\begin{array}{l}0.048999923 \\
0.039348027\end{array}$ & $\begin{array}{l}0.04721413 \\
0.037201222\end{array}$ \\
\hline & & 0.049762862 & & 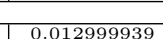 & $\begin{array}{r}\text { DTLZ5 } \\
0.008494638\end{array}$ & & 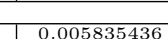 & & 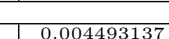 & \\
\hline M-CSEA & 0.424670314 & 0.03700648 & 0.01817932 & 0.013216065 & 0.009003176 & 0.007227901 & 0.006568781 & 0006156097 & 0.0044 & 50872 \\
\hline & & & & & & & & & & \\
\hline $\mathrm{M}-\mathrm{CS}$ & $\frac{0.02801940}{8.547355148}$ & $\frac{1.4802684075}{1.926878075}$ & $\begin{array}{l}0.15872128 \\
0.117556984\end{array}$ & $\begin{array}{l}0.018593501 \\
0.015819266\end{array}$ & $\begin{array}{l}0.0009888897 \\
0.00962453\end{array}$ & 0.006568246 & $\begin{array}{l}0.006343882 \\
0.004810618\end{array}$ & $\begin{array}{l}0.00060839191 \\
0.003727698\end{array}$ & 0.003479865 & $\begin{array}{l}0.00039032058 \\
0.003282058\end{array}$ \\
\hline & & & & & $\begin{array}{c}\text { DTLZ7 } \\
0.059149384\end{array}$ & & & & & \\
\hline M-CSEA & 8.948192414 & 1.211191478 & 0.068162702 & 0.050790774 & $\begin{array}{l}0.05149384 \\
0.042785891 \\
\end{array}$ & 0.038499199 & $\begin{array}{l}0.041051039 \\
0.035339474\end{array}$ & $\begin{array}{l}0.03564026 \\
0.032621292\end{array}$ & $\begin{array}{l}0.03012235 \\
0.031239453\end{array}$ & 0.030098333 \\
\hline & & & & & & & & & & \\
\hline & & $\frac{0.1}{0.1}$ & & & 0.111925885 & & & & & \\
\hline
\end{tabular}




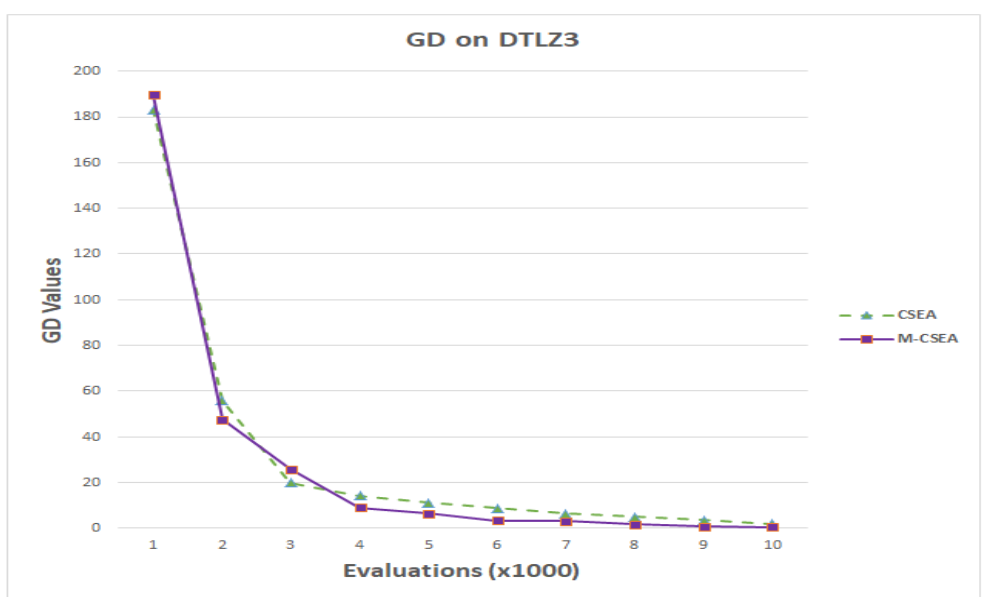

Fig. 14 The behavior of M-CSEA and CSEA on DTLZ3(GD)

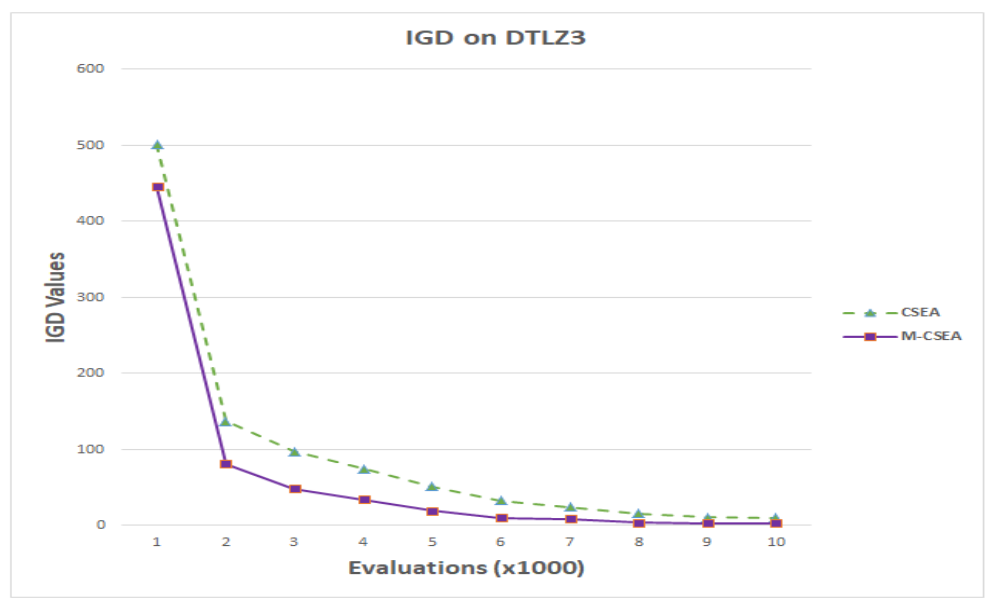

Fig. 15 The behavior of M-CSEA and CSEA on DTLZ3 (IGD)

Analyzing and comparing the results, with 10000 evaluations in 30 independent runs, we found that M-CSEA with dynamic selection strategy gets more balance of convergence and diversity for the obtained population. In the early stages (at 4000 evaluations), it gets better in 5 problems with GD (DTLZ2, DTLZ3, DTLZ4, DTLZ5, DTLZ8) and 6 problems on IGD (DTLZ1, DTLZ2, DTLZ3, DTLZ4, DTLZ5, DTLZ8). During the generations (at 7000 evaluations), M-CSEA gets better in 4 problems on GD (DTLZ2, DTLZ3, DTLZ5, DTLZ8). It is also better in 6 problems on IGD (DTLZ1, DTLZ3, DTLZ4, DTLZ6, DTLZ7, DTLZ8). In the last stages (at 10000 evaluations), M-CSEA gets better in 6 problems on GD (DTLZ1, DTLZ2, DTLZ3, DTLZ5, 


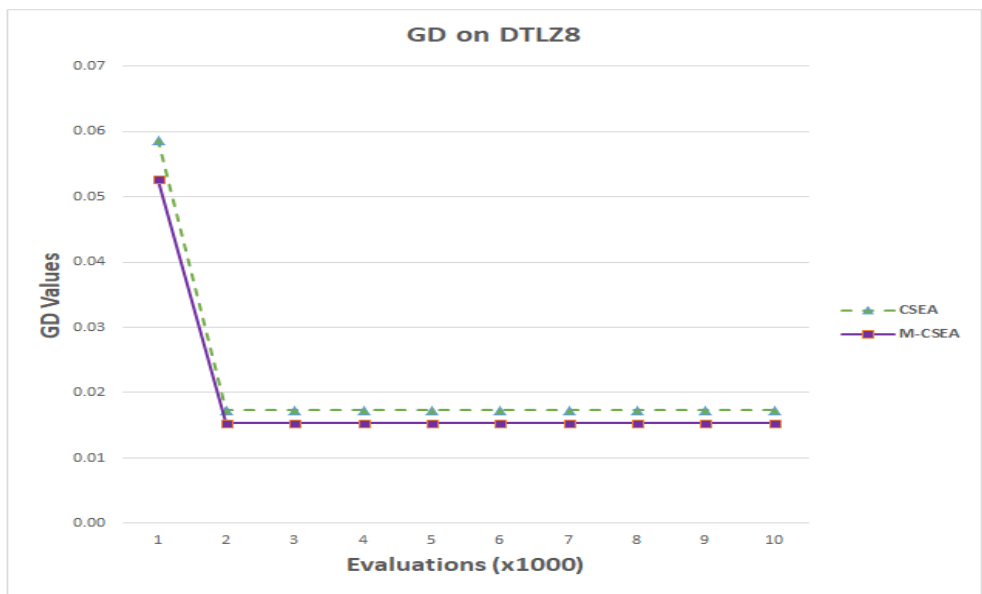

Fig. 16 The behavior of M-CSEA and CSEA on DTLZ8 (GD)

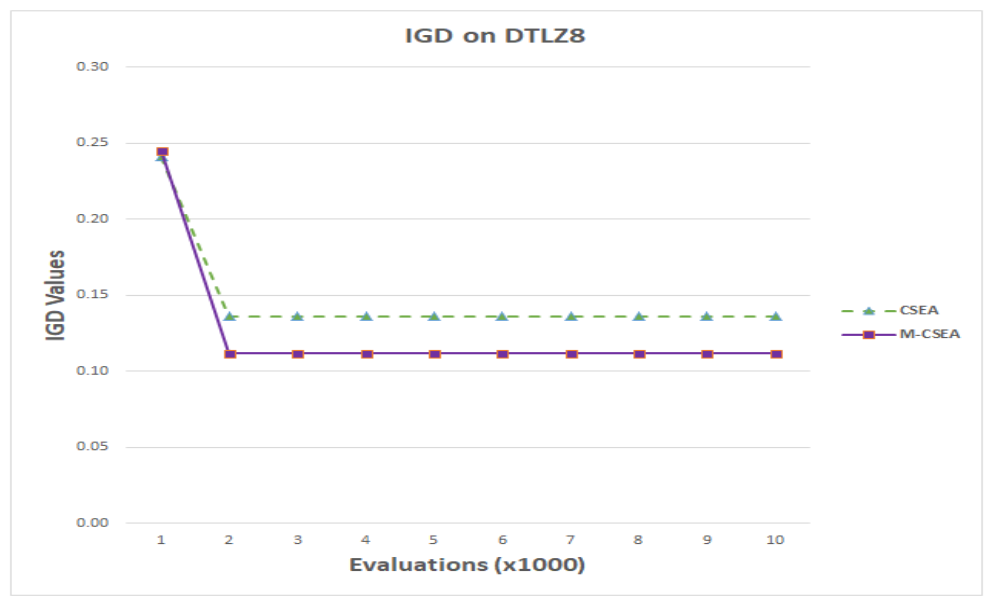

Fig. 17 The behavior of M-CSEA and CSEA on DTLZ8 (IGD)

DTLZ7, DTLZ8) and 6 problesm on IGD (DTLZ1, DTLZ3, DTLZ4, DTLZ6, DTLZ7, DTLZ8). In other problems, CSEA gets a bit better than M-CSEA. When considering the final results obtained after 10000 evaluations, M-CSEA achieves an improvement in the quality of the population achieved in terms of both convergence and diversity on most of the problems. However, for the DTLZ4 problem, the M-CSEA did not achieve an improvement for the CSEA. DTLZ4 is a problem with the characteristics of Pareto solutions non-uniformly distributed on Pareto Front, then there will be points with different density of solutions. The principle of the proposed adaptive guidance of convergence and diversity towards solutions that converge, but spread uniformly over Pareto Front, does not take into account the problem of density of each region on the objective space, especially on the surface of Pareto Front. Therefore, this 
can be a difficulty in adaptive adjustment to improve the convergence and diversity quality of M-CSEA.

In addition to a significant improvement in the convergence quality and diversity of the obtained solutions as well as maintaining a balance between exploitation and exploration of the evolutionary process, the proposed method helps reduce the computation time of surrogate-assisted multi-objective algorithms, especially suitable for solving expensive problems.

Through the method and the new the selection strategy on the adaptive number of reference solutions, which implements the optimal optimization algorithm using general surrogate functions, selection of reference solutions for sampling in machine learning such as the FNNs. The use of the response parameter is to assess the quality of the population and how many reference solutions should form the boundary of the classification. The results using dynamic selection strategy by the $K$ parameter demonstrate the impact of the time process, the current convergence quality of the population, and the characteristics of the problem on maintaining the equilibrium of convergence and diversity quality of the population, through ensuring a balance between exploration and exploitation process of evolution. The effectiveness of adaptive selection depends on the quality and effectiveness of the previous stage, proving that a timely adjustment maintains a balance between exploration and exploitation is needed. However, from the results of some problems, CSEA has better quality, although not large, it also poses challenges such as stability, sustainability, random factors course, etc. Those factors influence the choice of parameters appropriately or guide evolution to better efficiency. The robustness of the algorithm will be improved by reducing the noises with the dynamic selection strategy.

In future studies, we will continue to investigate the factors affecting the improvement of the quality of convergence and diversity, the ability to explore and exploit of the algorithm such as: discontinuous characteristics of Pareto Front, solution density on Pareto Front,... to further improve the proposal with better results.

\section{Experiments and Discussion}

In this section, we use the two enhanced algorithms proposed above to confirm the proposed model of the planning problem for the medical workforce to perform social tasks and the guidance technique.

\subsection{Experimental Setup}

The well-known MOEA Toolbox PlatEMO [16] with MatLab 2020b was used for the experiment. To compare the effectiveness we use the HV performance 
matrix. The experimental PC with configuration: HP ProLiant DL380; CPU: 2xIntel Xeon Silver 4110 Processor with 11M cache, 2.10GHz; RAM: 4x31GB PC4-21300; HDD: 4x1TB 7200 rpm SATA 6Gb/s.

The parameter settings:

- Number of variables: $n=12$ or $n=30$.

- Number of objectives: $m=3$.

- Population size: $N_{p}=100$.

- Number of Independence run: 10.

- Maximum origin fitness evaluations: $F E_{\max }=1000$

Experimental Datasets

Dataset 1 (12-task dataset): Working plan for 12 social tasks with parameters: $n=12 ; R_{1}=80 ; R_{2}=20 ; \alpha=0.1$, the task's attributes in table 7

Table 6 Dataset 1.

\begin{tabular}{|c|c|c|c|c|c|}
\hline$a_{i}$ & $d_{i}$ & $r_{1 i}$ & $r_{2 i}$ & $e_{i}$ & $P_{i}$ \\
\hline 1 & 16 & 24 & 3 & 3 & 0 \\
\hline 2 & 4 & 56 & 12 & 5 & 0 \\
\hline 3 & 12 & 30 & 5 & 8 & 0 \\
\hline 4 & 10 & 15 & 10 & 6 & 0 \\
\hline 5 & 8 & 25 & 2 & 4 & 1,2 \\
\hline 6 & 13 & 36 & 6 & 7 & 2,3 \\
\hline 7 & 15 & 22 & 10 & 5 & 4 \\
\hline 8 & 6 & 22 & 10 & 5 & 4 \\
\hline 9 & 14 & 16 & 4 & 10 & 5,6 \\
\hline 10 & 9 & 32 & 9 & 3 & 6,7 \\
\hline 11 & 7 & 18 & 11 & 8 & 7,8 \\
\hline 12 & 11 & 28 & 7 & 6 & 8 \\
\hline
\end{tabular}

Dataset 2 (30-task dataset): Working plan for 30 social tasks with parameters: $n=30 ; R_{1}=120 ; R_{2}=25 ; \alpha=0.1$, the task's attributes in table 7

4.2 Results and discussion

\subsubsection{Archived solutions}

The results of testing the working planning problem for each algorithms on each dataset are shown in the Figure 18 to Fig. 21:

\subsubsection{Results on $\mathrm{HV}$}

The results of the HV (Hypervolume) [18] performance metric value are reported to compare and evaluate the algorithms with each other and presented in Table 8. The HV measures bold values perform better. 
Table 7 Dataset 2

\begin{tabular}{|c|c|c|c|c|c|}
\hline$a_{i}$ & $d_{i}$ & $r_{1 i}$ & $r_{2 i}$ & $e_{i}$ & $P_{i}$ \\
\hline 1 & 16 & 24 & 3 & 3 & 0 \\
\hline 2 & 4 & 56 & 12 & 5 & 0 \\
\hline 3 & 12 & 30 & 5 & 8 & 0 \\
\hline 4 & 10 & 15 & 6 & 6 & 0 \\
\hline 5 & 8 & 25 & 7 & 4 & 0 \\
\hline 6 & 13 & 36 & 6 & 7 & 0 \\
\hline 7 & 15 & 12 & 3 & 9 & 1 \\
\hline 8 & 6 & 22 & 5 & 5 & 1 \\
\hline 9 & 14 & 16 & 3 & 10 & 2,3 \\
\hline 10 & 9 & 32 & 10 & 3 & 4,5 \\
\hline 11 & 7 & 18 & 5 & 8 & 5 \\
\hline 12 & 11 & 28 & 8 & 6 & 6 \\
\hline 13 & 8 & 22 & 4 & 5 & 7 \\
\hline 14 & 15 & 19 & 6 & 9 & 8,9 \\
\hline 15 & 9 & 28 & 9 & 7 & 10 \\
\hline 16 & 7 & 25 & 5 & 7 & 10 \\
\hline 17 & 5 & 18 & 4 & 5 & 11,12 \\
\hline 18 & 11 & 33 & 7 & 8 & 11 \\
\hline 19 & 13 & 24 & 8 & 3 & 13,14 \\
\hline 20 & 6 & 30 & 6 & 4 & 14 \\
\hline 21 & 10 & 14 & 9 & 10 & 15,16 \\
\hline 22 & 9 & 28 & 7 & 6 & 16 \\
\hline 23 & 6 & 23 & 5 & 5 & 17 \\
\hline 24 & 7 & 20 & 2 & 6 & 18 \\
\hline 25 & 12 & 17 & 4 & 7 & 19 \\
\hline 26 & 5 & 27 & 6 & 9 & 20 \\
\hline 27 & 4 & 30 & 8 & 8 & 20,21 \\
\hline 28 & 12 & 24 & 7 & 10 & 22,23 \\
\hline 29 & 15 & 16 & 3 & 4 & 23 \\
\hline 30 & 8 & 18 & 2 & 6 & 24 \\
\hline & & & & \\
& & & \\
\hline
\end{tabular}
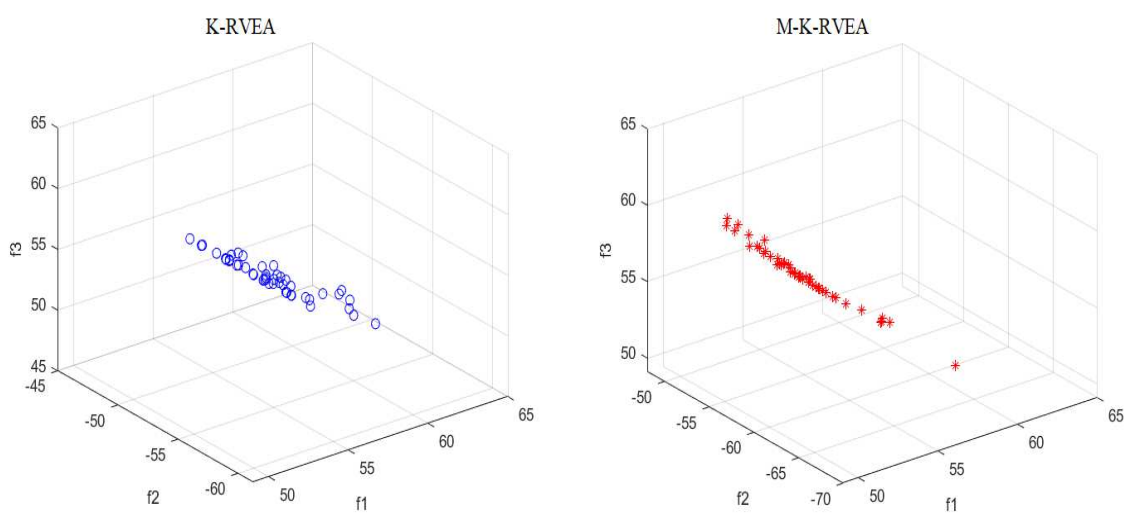

Fig. 18 The solution set on 12-task dataset (K-RVEA and M-K-RVEA).

\subsubsection{Discussion}

From the results of the 4 algorithms for the problem of planning work for social tasks, the solution set obtained using the improved algorithms (right) 

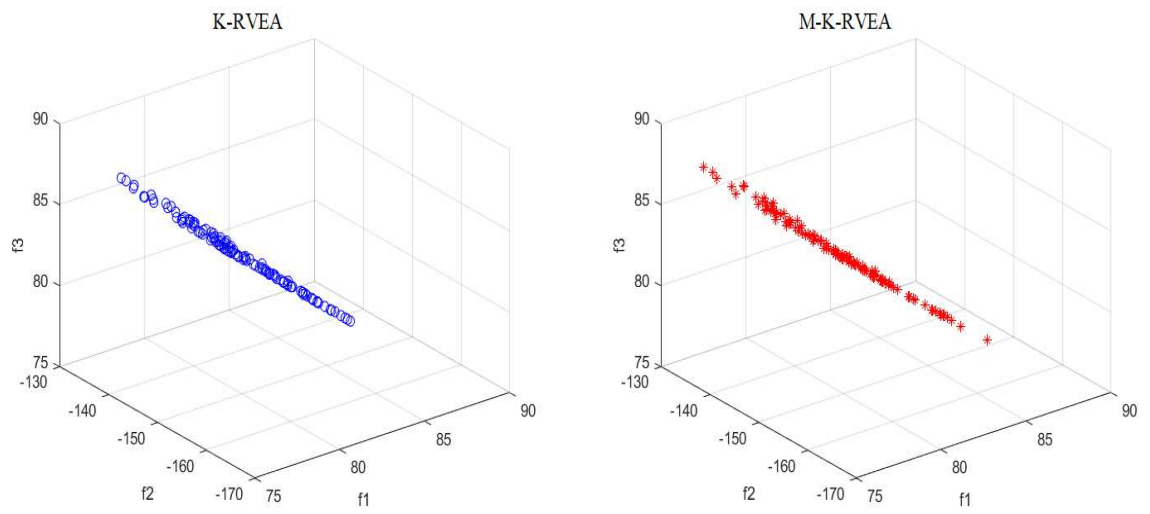

Fig. 19 The solution set on 30-task dataset (K-RVEA and M-K-RVEA).
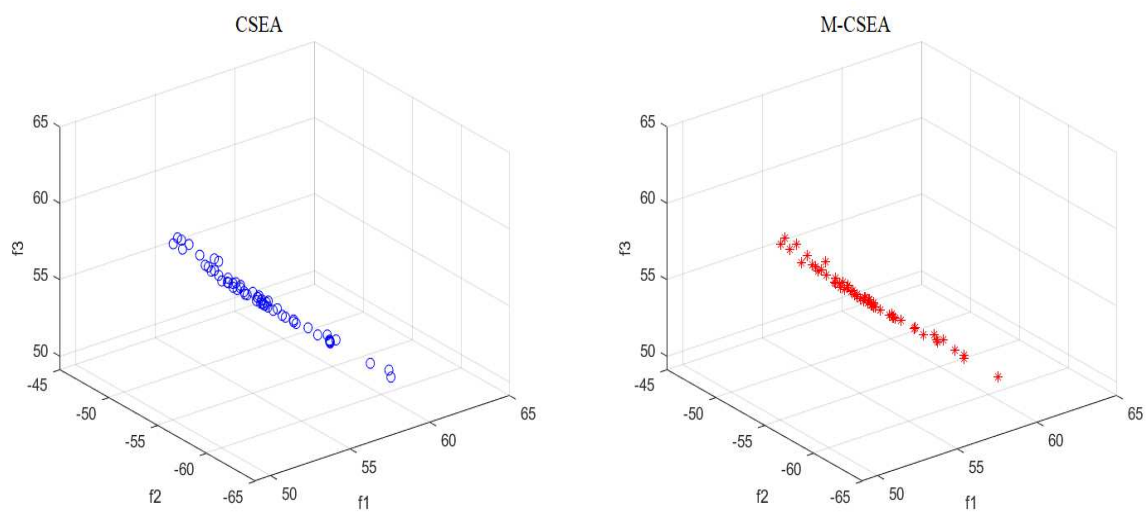

Fig. 20 The solution set on 12-task dataset (CSEA and M-CSEA).

outperforms the original algorithms (left) see that. The solution set obtained by the improved algorithms has a uniform distribution on the Pareto layer, which means that with the guidance technique, the algorithm has better diversity results. Additionally, through the evaluation results using the $\mathrm{HV}$ value. Along with the test results on the sample problem and the results obtained through experimenting with the real problem, the problem of work planning for social tasks (identified as one of the most expensive problems in the world, a noisy problem), experiments proves the factors affecting multi-objective evolutionary algorithms using surrogate functions. The automatic assessment (automatic guidance) is exceptionally efficient in achieving the main purposes, which includes improving the quality of convergence and diversity, maintaining a balance between the local exploration and global exploitation of evolution, suitable and effective to apply in solving difficult, expensive, and noisy problems in practice. 

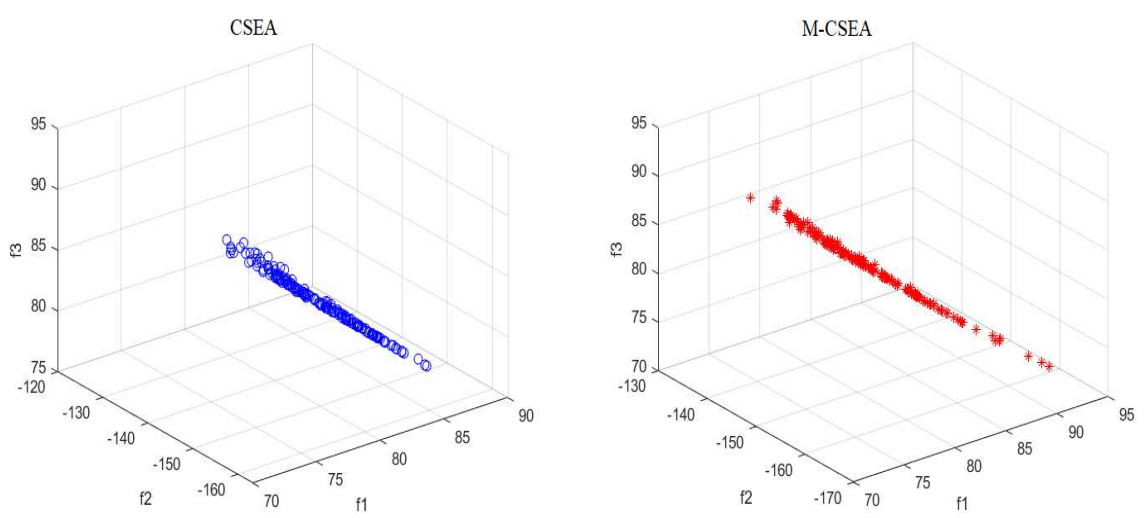

Fig. 21 The solution set on 30-task dataset (CSEA and M-CSEA).

Table 86 HV values of K-RVEA and M-K-RVEA, CSEA and M-CSEA on the two datasets.

\begin{tabular}{|l|l|l|l|l|}
\hline Run & K-RVEA & M-K-RVEA & CSEA & M-CSEA \\
\hline \multicolumn{5}{|c|}{ 12-task dataset } \\
\hline 1 & 0.273 & $\mathbf{0 . 2 7 8}$ & $\mathbf{0 . 2 7 6}$ & 0.275 \\
\hline 2 & 0.275 & $\mathbf{0 . 2 9 6}$ & 0.294 & $\mathbf{0 . 3 0 4}$ \\
\hline 3 & 0.273 & $\mathbf{0 . 2 7 3}$ & 0.277 & $\mathbf{0 . 2 9 3}$ \\
\hline 4 & 0.275 & $\mathbf{0 . 2 7 5}$ & $\mathbf{0 . 2 7 7}$ & 0.276 \\
\hline 5 & 0.274 & $\mathbf{0 . 2 9 3}$ & 0.275 & $\mathbf{0 . 2 9 1}$ \\
\hline 6 & $\mathbf{0 . 2 7 6}$ & 0.276 & 0.296 & $\mathbf{0 . 2 9 9}$ \\
\hline 7 & $\mathbf{0 . 2 8 5}$ & 0.275 & 0.275 & $\mathbf{0 . 3 0 4}$ \\
\hline 8 & 0.273 & $\mathbf{0 . 2 7 4}$ & 0.276 & $\mathbf{0 . 2 8 7}$ \\
\hline 9 & $\mathbf{0 . 2 7 5}$ & 0.273 & 0.276 & $\mathbf{0 . 2 9 3}$ \\
\hline 10 & 0.274 & $\mathbf{0 . 2 9 3}$ & 0.305 & 0.293 \\
\hline Average & 0.275 & $\mathbf{0 . 2 8 1}$ & 0.283 & $\mathbf{0 . 2 9 2}$ \\
\hline \multicolumn{5}{|l|}{30 -task dataset } \\
\hline 1 & 0.312 & $\mathbf{0 . 3 1 6}$ & 0.314 & $\mathbf{0 . 3 2 3}$ \\
\hline 2 & 0.313 & $\mathbf{0 . 3 2 9}$ & 0.322 & $\mathbf{0 . 3 4 9}$ \\
\hline 3 & $\mathbf{0 . 3 1 0}$ & 0.304 & 0.316 & $\mathbf{0 . 3 4 1}$ \\
\hline 4 & 0.303 & $\mathbf{0 . 3 0 3}$ & 0.313 & $\mathbf{0 . 3 2 3}$ \\
\hline 5 & 0.314 & $\mathbf{0 . 3 1 8}$ & $\mathbf{0 . 3 1 8}$ & 0.313 \\
\hline 6 & 0.313 & $\mathbf{0 . 3 1 8}$ & 0.313 & $\mathbf{0 . 3 1 5}$ \\
\hline 7 & 0.320 & $\mathbf{0 . 3 3 6}$ & 0.317 & $\mathbf{0 . 3 3 0}$ \\
\hline 8 & 0.313 & $\mathbf{0 . 3 1 3}$ & 0.308 & $\mathbf{0 . 3 2 0}$ \\
\hline 9 & 0.308 & $\mathbf{0 . 3 1 2}$ & $\mathbf{0 . 3 1 3}$ & 0.309 \\
\hline 10 & 0.311 & $\mathbf{0 . 3 2 2}$ & $\mathbf{0 . 3 2 3}$ & 0.313 \\
\hline Average & 0.312 & $\mathbf{0 . 3 1 7}$ & 0.316 & $\mathbf{0 . 3 2 3}$ \\
\hline
\end{tabular}

\section{Conclusion}

The problem of planning work for social tasks is difficult because of the effective requirements to solve the big problems of society. One important factor that determines the success of planning for social tasks is the effectiveness, redundancy, readiness to improvise and adjust the plan based on the reserve force. The organization of the reserve force depends on the effective use of hu- 
man resources in the organization and implementation of the plan from time to time.

From the required characteristics of planning problems for social tasks, the paper solves the planning optimization problem on a multi-objective approach with 3 main objectives: 1) minimize total time, 2) maximize of total effectiveness, and 3) minimize the average use of human resources with constraint functions.

The paper also proposes multi-objective optimization algorithms using surrogate models. We investigated the requirements for using surrogate models such as sample selection, frequency of using surrogate functions, machine learning process, the training process. Also, we considered the noisy evolutionary environment, factors affecting the ability of exploration and exploitation of algorithms using typical representative models. The paper also proposed a guidance technique for the algorithms by building an adaptive frequency adjustment mechanism using the original function on K-RVEA and adjusting sampling for the training process of the surrogate model in CSEA with consideration of factors in the evolutionary process. Therefore, the proposed algorithms improve the quality of solutions and the balance between local exploration and global exploitation of the evolutionary process. From the results of testing the model for the problem of planning social tasks using the enhanced multi-objective evolutionary algorithms, comparing and analyzing the set of solutions achieved, we confirmed the feasibility of the proposed problem model and the effectiveness of the guidance technique for algorithms using surrogate models for expensive problems, especially difficult and noisy problems in the real world.

The problem of planning work for work teams to perform social tasks is difficult with many impacts and constraints. The study focuses on sustainability, prevention, and adaptation to changes in the implementation process of modeling multi-objective problems. However, to respond to real-world problems, for example, the problem of planning for working teams to prevent and treat the current COVID-19 pandemic, it is necessary to investigate more requirements and constraints as well as inheriting research results in the paper. Also, this subject is positioned for further research in the future. 


\section{Declarations}

Funding: No funding.

Conflicts of interest/Competing interests: No

Availability of data and material (data transparency): Yes Code availability (software application or custom code): Yes Authors' contributions:

1. Proposed a model using a multi-objective optimization algorithm (with 03 objectives) for the problem of planning for social forces, especially planning for social forces in the COVID-19 epidemic prevention strategy.

2. Proposed techniques to improve two multi-objective optimization algorithms using surrogate models CSEA and KRVEA and use them in experiments to prove the proposed model.

Additional declarations for articles in life science journals that report the results of studies involving humans and/or animals: No. Ethics approval (include appropriate approvals or waivers): Yes.

Consent to participate (include appropriate statements): Yes.

Consent for publication (include appropriate statements): Yes. 


\section{References}

1. Bakhtavar, E., Prabatha, T., Karunathilake, H., Sadiq, R., Hewage, K.: Assessment of renewable energy-based strategies for net-zero energy communities: A planning model using multi-objective goal programming. Journal of Cleaner Production 272, 122886 (2020)

2. Cai, C., Chen, J., Xi, M., Tao, Y., Deng, Z.: Multi-objective planning of distributed photovoltaic power generation based on multi-attribute decision making theory. IEEE Access 8, 223021-223029 (2020)

3. Chugh, T.: Handling expensive multi-objective optimization problems with evolutionary algorithms. University of Jyvaskyla (2017)

4. Chugh, T., Jin, Y., Miettinen, K., Hakanen, J., Sindhya, K.: K-rvea: a kriging-assisted evolutionary algorithm for many-objective optimization. Reports of the Department of Mathematical Information Technology, Series B, Scientific Computing no. B 2 (2016)

5. Deb, K., Thiele, L., Laumanns, M., Zitzler, E.: Scalable test problems for evolutionary multi-objective optimization. In: Evolutionary Multi-objective Optimization, pp. 105145. Springer, London (2005)

6. Diaz-Manriquez, A., Toscano, G., Barron-Zambrano, J.H., Tello-Leal, E.: A multiobjective optimization model for project scheduling with time-varying resource requirements and capacities. Computational Intelligence and Neuroscience 2016 (2016)

7. Ganguly, S.: Multi-objective distributed generation penetration planning with load model using particle swarm optimization. Decision Making: Applications in Management and Engineering 3(1), 30-42 (2020)

8. Gene, L., Steven, C.: Multi-objective evolutionary neural network to predict graduation success at the united states military academy. Procedia Computer Science 140, 196-205 (2018)

9. Habibi, F., Farnaz, B., Seyed, J.S.: A multi-objective optimization model for project scheduling with time-varying resource requirements and capacities. Journal of Industrial and Systems Engineering 10(Special Issue on Scheduling), 92-118 (2017)

10. Hu, X., Zhang, H., Chen, D., Li, Y., Wang, L., Zhang, F., Cheng, H.: Multi-objective planning for integrated energy systems considering both exergy efficiency and economy. Energy 197, 117155 (2020)

11. Lo, W.C., Lu, C.H., Chou, Y.C.: Application of multicriteria decision making and multiobjective planning methods for evaluating metropolitan parks in terms of budget and benefits. Mathematics 8(8), 1304 (2020)

12. Myers, R.H., Montgomery, D., Anderson-Cook, C.M.: Response surface methodology: process and product optimization using designed experiments. John Wiley \& Sons (2016)

13. Pan, L., He, C., Tian, Y., Wang, H., Zhang, X., Jin, Y.: A classification-based surrogateassisted evolutionary algorithm for expensive many-objective optimization. IEEE Transactions on Evolutionary Computation 23(1), 74-88 (2018)

14. Raafat, E., Mona, S., Hesham, E., Gamal, N.: Solving resource-constrained project scheduling problem using genetic algorithm. Journal Of Al Azhar University Engineering Sector 12(42), 187-198 (2017)

15. Soleymani, F., Barfeie, M., Haghani, F.K.: Inverse multi-quadric rbf for computing the weights of fd method: Application to american options. Communications in Nonlinear Science and Numerical Simulation 64, 74-88 (2018)

16. Tian, Y., Cheng, R., Zhang, X., Jin, Y.: Platemo: A matlab platform for evolutionary multi-objective optimization [educational forum]. IEEE Computational Intelligence Magazine 12(4), 73-87 (2017)

17. Vapnik, V.: The nature of statistical learning theory. Springer Science \& Business Media (2013)

18. Zitzler, E., Thiele, L.: Multiobjective evolutionary algorithms: a comparative case study and the strength pareto approach. IEEE transactions on Evolutionary Computation 3(4), 257-271 (1999)

19. Zitzler, E., Thiele, L., Deb, K.: Comparision of multiobjective evolutionary algorithms: Emprical results. Evolutionary Computation 8(1), 173-195 (2000) 